\title{
OsXCl $\left(\mathrm{PPh}_{3}\right)_{3}(\mathrm{X}=\mathrm{H}, \mathrm{Cl})$ 与炔丙基氯反应合成烯基卡拜配合物及 膦配体调控的锇-氢卡拜向卡宾配合物的转化
}

\author{
杨玉 蔡 涛 温庭斌* \\ (厦门大学化学化工学院化学系 厦门 361005)
}

\begin{abstract}
摘要 简单的锇起始物 $\mathrm{OsHCl}\left(\mathrm{PPh}_{3}\right)_{3}$ (1)或 $\mathrm{OsCl}_{2}\left(\mathrm{PPh}_{3}\right)_{3}$ (2)与 3-氯-3-甲基-1-丁炔反应可分别得到锇的烯基卡拜配合物 $\mathrm{OsHCl}_{2}\left(\equiv \mathrm{CCH}=\mathrm{CMe}_{2}\right)\left(\mathrm{PPh}_{3}\right)_{2}(\mathbf{3})$ 和 $\mathrm{OsCl}_{3}\left(\equiv \mathrm{CCH}=\mathrm{CMe}_{2}\right)\left(\mathrm{PPh}_{3}\right)_{2}$ (4). 化合物 $\mathbf{3}$ 与二苯基(苯乙炔基)膦反应可转化为三 个二苯基(苯乙炔基)膦配位的锇烯基卡宾配合物 $\mathrm{OsCl}_{2}\left(=\mathrm{CHCH}=\mathrm{CMe}_{2}\right)\left(\mathrm{Ph}_{2} \mathrm{PC} \equiv \mathrm{CPh}\right)_{3}$ (5), 而化合物 4 与二苯基(苯乙 炔基)膦反应得到膦配体取代产物 $\mathrm{OsCl}_{3}$ ( $\left.\equiv \mathrm{CCH}=\mathrm{CMe}_{2}\right)\left(\mathrm{Ph}_{2} \mathrm{PC} \equiv \mathrm{CPh}\right)_{2}$ (6). 二苯基(苯乙炔基)膦配体较弱的供电子能 力及较小的空间位阻是促成化合物 3 发生锇上的原子氢配体到卡拜碳的 1,2-迁移而转化为卡宾配合物 5 的原因.
\end{abstract}

关键词 锇; 卡拜; 卡宾; 二苯基(苯乙炔基)膦; 炔丙基氯

\section{Alkenylcarbyne Complexes Derived from the Reactions of OsXCl- $\left(\mathrm{PPh}_{3}\right)_{3}(\mathrm{X}=\mathrm{H}, \mathrm{Cl})$ with Propargyl Chloride and Phosphine Ligand- Controlled Transformation of Hydride-Carbyne to Carbene}

\author{
Yang, Yu Cai, Tao Wen, Tingbin* \\ (Department of Chemistry, College of Chemistry and Chemical Engineering, Xiamen University, Xiamen 361005)
}

\begin{abstract}
Reactions of the simple osmium precursors $\mathrm{OsHCl}\left(\mathrm{PPh}_{3}\right)_{3}(\mathbf{1})$ or $\mathrm{OsCl}_{2}\left(\mathrm{PPh}_{3}\right)_{3}$ (2) with 3-chloro-3-methylbut-1-yne afforded $\mathrm{OsHCl}_{2}\left(\equiv \mathrm{CCH}=\mathrm{CMe}_{2}\right)\left(\mathrm{PPh}_{3}\right)_{2}$ (3) or $\mathrm{OsCl}_{3}\left(\equiv \mathrm{CCH}=\mathrm{CMe}_{2}\right)\left(\mathrm{PPh}_{3}\right)_{2}(4)$, respectively. Treatment of 3 with $\mathrm{Ph}_{2} \mathrm{PC} \equiv$ $\mathrm{CPh}$ led to the formation of the tris-diphenyl(phenylethynyl)phosphine alkenylcarbene complex $\mathrm{OsCl}_{2}\left(=\mathrm{CHCH}=\mathrm{CMe}_{2}\right)\left(\mathrm{Ph}_{2} \mathrm{PC}\right.$ $\equiv \mathrm{CPh})_{3}(5)$, while complex 4 underwent simple phosphine ligand substitution with $\mathrm{Ph}_{2} \mathrm{PC} \equiv \mathrm{CPh}$ to give $\mathrm{OsCl}{ }_{3} \equiv \mathrm{CCH}=$ $\left.\mathrm{CMe}_{2}\right)\left(\mathrm{Ph}_{2} \mathrm{PC} \equiv \mathrm{CPh}\right)_{2}(6)$. Presumably, upon the phosphine ligand substitution of $\mathrm{PPh}_{3}$ in 3 by $\mathrm{Ph}_{2} \mathrm{PC} \equiv \mathrm{CPh}$, the relatively electron-poor nature of the later decreased the electron density of the osmium center, and thus promoted the transformation of the hydride-carbyne to carbene via the 1,2-shift of the hydride ligand from Os to the carbyne carbon, which was then further facilitated by the coordination of a third less bulky $\mathrm{Ph}_{2} \mathrm{PC} \equiv \mathrm{CPh}$ ligand to the osmium center to give the stable $18 \mathrm{e}$ product 5 .

Keywords osmium; carbyne; carbene; diphenyl(phenylethynyl)phosphine; propargyl chloride
\end{abstract}

过渡金属卡拜配合物 $\left(\mathrm{L}_{n} \mathrm{M} \equiv \mathrm{CR}\right.$ ，亦即含金属-碳参 键的配合物)因具有特殊的性质以及在有机合成和催化 反应中的重要应用而引起了人们广泛的研究兴趣 ${ }^{[1]}$, 特 别是以卡拜配合物为催化活性物种的炔烃复分解反应 现已逐渐成为有机合成中的一个重要工具反应 ${ }^{[2]}$. 然而, 目前具有炔烃复分解活性的催化剂还仅限于前过渡金 属(主要为 Schrock 型的钿、铇的卡拜配合物或其前 体 $)^{[2,3]}$. 作为目前研究得最多的后过渡金属卡拜配合物,
锇卡拜也因其丰富的反应性质以及人们对探索后过渡 金属卡拜配合物的炔烃复分解活性的兴趣而越来越受 到关注 ${ }^{[4]}$ ，近年来涌现了相当多锇卡拜配合物的报道， 并发现了许多有趣的反应性质 ${ }^{[4 a, 4 b, 5 \sim 8]}$.

另一方面，卡拜配合物不论在结构、合成方法还是 在反应性质方面都和卡宾配合物存在着密切的关 系 $^{[1 \mathrm{~d}, 1 \mathrm{e}, 2 \mathrm{~b}, 9]}$ ，例如，文献报道了许多金属-氢卡拜配合物 $\mathrm{L}_{n} \mathrm{MH}(\equiv \mathrm{CR})$ 和卡宾配合物 $\mathrm{L}_{n} \mathrm{M}(=\mathrm{CHR})$ 之间转化的例

\footnotetext{
* Corresponding author. E-mail: chwtb@xmu.edu.cn

Received August 18, 2016; revised September 4, 2016; published online September 6, 2016.

Project supported by the National Basic Research Program of China (973 Program, No. 2012CB821600) and the National Natural Science Foundation of China (No. 21072161).

国家重点基础研究发展计划(973 计划, No. 2012CB821600)和国家自然科学基金(No. 21072161)资助项目.
} 
子, 一系列 $6 \sim 8$ 族的金属-氢卡拜配合物可以通过氢配 体从金属向卡拜 $\alpha$-碳的迁移而转化为卡宾 ${ }^{[10 ~ 13]}$, 卡宾 配合物也可经氢原子从卡宾 $\alpha$-碳向金属中心的 1,2 -氢迁 移转化为卡拜配合物, 但主要发生在第三过渡系的

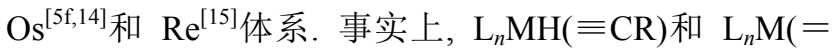
CHR)可看作一对氧化-还原异构体, 两者之间存在着平 衡, 而这个氧化-还原平衡的位置及相互转化的活化能 取决于金属中心的电子富足程度, 因此与金属中心本身 及配体都有关系 ${ }^{[4 a, 12]}$. 对于富电子金属中心的配合物, 金属还原性强，易被氧化而更倾向于形成含更多金属碳键的氧化异构体, 因而有利于生成配位饱和的 18 电 子产物 $\mathrm{L}_{n} \mathrm{MH}(\equiv \mathrm{CR})$, 反之则是倾向于生成还原异构体 $\mathrm{L}_{n} \mathrm{M}(=\mathrm{CHR})$. 在钉、锇体系中, $\mathrm{MCl}_{2}(=\mathrm{CHR})\left(\mathrm{PR}_{3}^{\prime}\right)_{2}$ 类 型的卡宾配合物与相应的金属一氢卡拜 $\mathrm{MHCl}_{2}(\equiv$ $\mathrm{CR})\left(\mathrm{PR}_{3}^{\prime}\right)_{2}(\mathrm{M}=\mathrm{Ru}, \mathrm{Os})$ 的情况很好地体现了这种趋势. 锇与钉虽为同一族过渡金属，但锇处于更下一个周期， 还原性比钓强, 因此, 在反应中更倾向于生成配位饱和 的氧化异构体锇一氢卡拜配合物 $\mathrm{OsHCl}_{2}(\equiv \mathrm{CR})$ $\left(\mathrm{PR}_{3}\right)_{2}{ }_{2}{ }^{[4 a, 12,16]}$, 而钉的类似反应则是生成其还原异构体 卡宾配合物 $\mathrm{RuCl}_{2}(=\mathrm{CHR})\left(\mathrm{PR}_{3}^{\prime}\right)_{2}{ }^{[16,17]}$. 正因如此, 报道 的锇一氢卡拜 $\mathrm{OsHCl}_{2}(\equiv \mathrm{CR})\left(\mathrm{PR}_{3}\right)_{2}{ }_{2}{ }^{[4 a, b, 5 f, 12,13 c, 16,17 \mathrm{c}, 18,19]}$ 和 钉卡宾 $\mathrm{RuCl}_{2}(=\mathrm{CHR})\left(\mathrm{PR}_{3}\right)_{2}{ }^{[17,20]}$ 的例子很多, 相应的五 配位的锇卡宾 $\mathrm{OsCl}_{2}(=\mathrm{CHR})\left(\mathrm{PR}_{3}^{\prime}\right)_{2}$ 却很少见 ${ }^{[5,, 21]}$. Esteruelas 小组 ${ }^{[4 a, 6 c]}$ 通过密度泛函理论(DFT) 计算发现, 六配位的锇-氢卡拜与五配位的锇卡宾在能量上是相近 的, 但是发生氢迁移的能垒太高, 可以通过改变配合物 中的辅助配体的电子性质来调控金属中心的电子密度, 从而控制两者之间的平衡, 实现两者的相互转化

在发展烯烃复分解钓卡宾催化剂的过程中, Grubbs 小组于 1997 年报道了 $\mathrm{RuH}(\mathrm{H})_{2} \mathrm{Cl}\left(\mathrm{PCy}_{3}\right)_{2}$ 与炔丙基氯 $\mathrm{HC} \equiv \mathrm{CC}(\mathrm{Cl}) \mathrm{R}^{1} \mathrm{R}^{2}$ 的反应, 发展了一个通过炔烃插入 $\mathrm{Ru}-\mathrm{H}$ 键再发生 $\gamma$-氯消除的机理合成一系列钓烯基卡 宾化合物 $\mathrm{RuCl}_{2}\left(=\mathrm{CHCH}=\mathrm{CR}^{1} \mathrm{R}^{2}\right)\left(\mathrm{PCy}_{3}\right)_{2}$ 的简便方 法 ${ }^{[22]}$. Hofmann ${ }^{[23]}$ 和 Fogg 小组 ${ }^{[24]}$ 随后也各自分别报道 了类似的 $\mathrm{RuHCl}\left(\mathrm{PPh}_{3}\right)_{3}$ 与 3-氯-3-甲基-1-丁炔 $[\mathrm{HC} \equiv$ $\mathrm{CC}(\mathrm{Cl}) \mathrm{Me}_{2}$ ] 反应，很容易地合成了钓烯基卡宾 $\mathrm{RuCl}_{2}(=$ $\left.\mathrm{CHCH}=\mathrm{CMe}_{2}\right)\left(\mathrm{PPh}_{3}\right)_{2}$. 与此相关, Werner 等 ${ }^{[25]} 1999$ 年曾报道了 $\mathrm{OsH}_{3} \mathrm{Cl}\left(\mathrm{PCy}_{3}\right)_{2}$ 与 3-氯-3-甲基-1-丁炔的反 应，通过原位核磁检测到 $-40{ }^{\circ} \mathrm{C}$ 下反应生成了分子氢 配位的烯基卡宾配合物 $\mathrm{Os}\left(\mathrm{H}_{2}\right) \mathrm{Cl}_{2}(=\mathrm{CHCH}=$ $\left.\mathrm{CMe}_{2}\right)\left(\mathrm{PCy}_{3}\right)_{2}$ 和烯基卡拜配合物 $\mathrm{OsHCl}_{2}(\equiv \mathrm{CCH}=$ $\left.\mathrm{CMe}_{2}\right)\left(\mathrm{PCy}_{3}\right)_{2}$ 的混合物, 但前者非常不稳定, 室温下易 释放出配位的氢分子并在 $1 \mathrm{~h}$ 内就完全转化为后者. 鉴 于 $\mathrm{OsHCl}\left(\mathrm{PPh}_{3}\right)_{3}{ }^{[26]}$ 是比 $\mathrm{OsH}_{3} \mathrm{Cl}\left(\mathrm{PCy}_{3}\right)_{2}$ 更为简便易得的 锇原料, 以及前面所述钉、锇体系反应性质的区别, 本
文研究了 $\mathrm{OsHCl}\left(\mathrm{PPh}_{3}\right)_{3}$ 与 $\mathrm{HC} \equiv \mathrm{CC}(\mathrm{Cl}) \mathrm{Me}_{2}$ 的反应, 发 现可简便地制备锇一氢烯基卡拜配合物 $\mathrm{OsHCl}_{2}$ ( $\left.\mathrm{CCH}=\mathrm{CMe}_{2}\right)\left(\mathrm{PPh}_{3}\right)_{2}$ (3), 将反应拓展到 $\mathrm{OsCl}_{2}\left(\mathrm{PPh}_{3}\right)_{3}$ 与 $\mathrm{HC} \equiv \mathrm{CC}(\mathrm{Cl}) \mathrm{Me}_{2}$ 的反应，则可方便地制备三氯配位的 锇烯基卡拜配合物 $\mathrm{OsCl}_{3}\left(\equiv \mathrm{CCH}=\mathrm{CMe}_{2}\right)\left(\mathrm{PPh}_{3}\right)_{2}(4)$.

此外, 有机膦配体可通过改变取代基来调节其电子 性质和空间位阻，从而调控络合物的反应性质.二苯基 (苯乙炔基) 膦 $\left(\mathrm{Ph}_{2} \mathrm{PC} \equiv \mathrm{CPh}\right)$ 与 $\mathrm{PPh}_{3} 、 \mathrm{PCy}_{3} 、 \mathrm{P}(i-\mathrm{Pr})_{3}$ 等膦 配体相比, 具有体积小、给电子能力相对较弱的特点, 而且迄今为止还未见炔基膦配位的锇卡拜配合物的报 道, 因此, 本文也研究了 $\mathrm{OsXCl}_{2}\left(\equiv \mathrm{CCH}=\mathrm{CMe}_{2}\right)\left(\mathrm{PPh}_{3}\right)_{2}$ $(\mathrm{X}=\mathrm{H}, 3 ; \mathrm{X}=\mathrm{Cl}, 4$ )与二苯基(苯乙炔基)膦的反应，发现 前者发生了膦配体取代促成的锇上的原子氢配体到卡 拜 $\alpha$-碳的 1,2-迁移而转化为 3 个二苯基(苯乙炔基)膦配 位的烯基卡宾配合物 $\mathrm{OsCl}_{2}\left(=\mathrm{CHCH}=\mathrm{CMe}_{2}\right)\left(\mathrm{Ph}_{2} \mathrm{PC} \equiv\right.$ $\mathrm{CPh})_{3}(\mathbf{5})$, 后者仅发生膦配体取代生成双二苯基(苯乙 炔基)膦配位的锇烯基卡拜配合物 $\mathrm{OsCl}_{3}$ ( $\equiv \mathrm{CCH}=$ $\left.\mathrm{CMe}_{2}\right)\left(\mathrm{Ph}_{2} \mathrm{PC} \equiv \mathrm{CPh}\right)_{2}$ (6).

\section{1 结果与讨论}

\section{$1.1 \mathrm{OsXCl}\left(\mathrm{PPh}_{3}\right)_{3}(\mathrm{X}=\mathrm{H}, \mathrm{Cl})$ 与 3-氯-3-甲基-1-丁炔的 反应}

钉氢配合物 $\left.\mathrm{RuH}(\mathrm{H})_{2} \mathrm{Cl}(\mathrm{PCy})_{2}\right)_{2}$ 或 $\mathrm{RuHCl}\left(\mathrm{PPh}_{3}\right)_{3}$ 与炔 丙基氯反应可简便地制备钉烯基卡宾化合物 $\mathrm{RuCl}_{2}(=$ $\left.\mathrm{CHCH}=\mathrm{CR}_{1} \mathrm{R}_{2}\right)\left(\mathrm{PR}_{3}\right)_{2}{ }^{[22 \sim 24]}$, 而锇氢配合物 $\mathrm{OsH}_{3} \mathrm{Cl}$ $\left(\mathrm{PCy}_{3}\right)_{2}$ 与 3-氯-3-甲基-1-丁炔反应则是生成锇-氢烯基 卡拜 $\mathrm{OsHCl}_{2}\left(\equiv \mathrm{CCH}=\mathrm{CMe}_{2}\right)\left(\mathrm{PCy}_{3}\right)_{2}{ }^{[25]}$, 因此, 我们考 察了更为简单易得的锇原料 $\mathrm{OsHCl}\left(\mathrm{PPh}_{3}\right)_{3}$ 与 3-氯-3-甲 基-1-丁炔的反应. 室温下, $\mathrm{OsHCl}\left(\mathrm{PPh}_{3}\right)_{3}$ 和 3-氯-3-甲基1-丁炔的物质的量比为 $1: 1.2$ 时, 在甲苯中即可发生反 应, 且有棕色固体产物析出, $4 \mathrm{~h}$ 后将反应液静置, 经 ${ }^{31} \mathrm{P}\left\{{ }^{1} \mathrm{H}\right\} \mathrm{NMR}$ 检测上清液发现, 原料的信号消失, 除了 在 $\delta-5.3$ 附近有解离的 $\mathrm{PPh}_{3}$ 信号外, 在 $\delta 6.0$ 处有新的 单峰信号生成, 将上述反应液浓缩后, 经过滤、洗涤分 离得到锇 - 氢烯基卡拜配合物 $\mathrm{OsHCl}_{2}(\equiv \mathrm{CCH}=$ $\left.\mathrm{CMe}_{2}\right)\left(\mathrm{PPh}_{3}\right)_{2}(3)$, 产率 80\% (Scheme 1).

化合物 3 经过了 $\mathrm{X}$ 射线单晶衍射、核磁及元素分析 等完整表征, 晶体结构如图 1 所示, 与已报道锇氢烯基 卡拜配合物 $\mathrm{OsHCl}_{2}\left(\equiv \mathrm{CCH}=\mathrm{CMe}_{2}\right)\left(\mathrm{PCy}_{3}\right)_{2}$ 相似 ${ }^{[25]}$. 锇 中心的配位构型可以看成一个扭曲的八面体，两个 $\mathrm{PPh}_{3}$ 配体反式排列占据轴向位置, 而两个氯配体呈顺 式，并与氢配体以及卡拜碳占据赤道面. 化合物 3 的 $\mathrm{Os}(1)-\mathrm{C}(1)$ 的键长为 $0.1734(4) \mathrm{nm}$, 是典型的锇卡拜化 合物中 $\mathrm{Os}-\mathrm{C}$ 参键的键长 ${ }^{[4,5,5]}, \mathrm{C}(2)-\mathrm{C}(3)$ 的键长为 


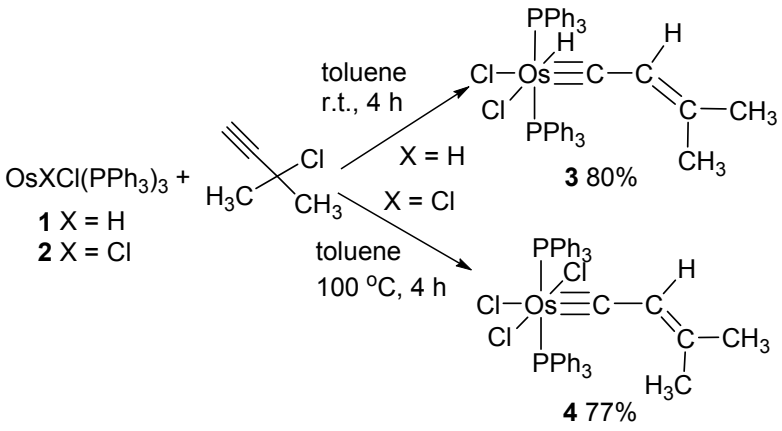

图式 $1 \mathrm{OsXCl}\left(\mathrm{PPh}_{3}\right)_{3}(\mathrm{X}=\mathrm{H}, \mathrm{Cl})$ 与 3-氯-3-甲基-1-丁炔的反 应

Scheme 1 Reactions of $\mathrm{OsXCl}\left(\mathrm{PPh}_{3}\right)_{3}(\mathrm{X}=\mathrm{H}, \mathrm{Cl})$ with 3-chloro-3-methyl-but-1-yne

$0.1352(5) \mathrm{nm}$, 是典型双键的键长, $\mathrm{C}(1)-\mathrm{C}(2)$ 的键长为 $0.1422(5) \mathrm{nm}$, 为 $\mathrm{C}(\mathrm{sp})-\mathrm{C}\left(\mathrm{sp}^{2}\right)$ 单键的键长, 因此是烯 基卡拜的结构, 与其他一些已报道的锇烯基卡拜化合物

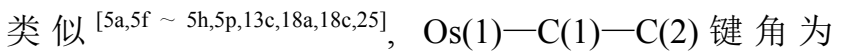
166.7(4) $)^{\circ}$, 接近线型结构. 有些轻微的弯曲, 在一些已 报道的锇卡拜化合物中也有相似情况 ${ }^{[4 b, 5 f, 5 g, 5 p, 14 a, 27]}$.

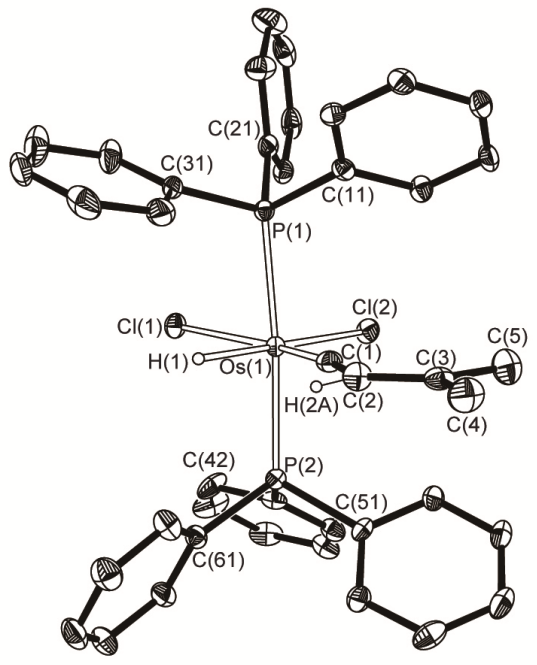

图 $1 \mathrm{OsHCl}_{2}\left(\equiv \mathrm{CCH}=\mathrm{CMe}_{2}\right)\left(\mathrm{PPh}_{3}\right)_{2}(3)$ 的晶体结构图

Figure 1 Molecular structure of $\mathrm{OsHCl}_{2}\left(\equiv \mathrm{CCH}=\mathrm{CMe}_{2}\right)$ $\left(\mathrm{PPh}_{3}\right)_{2}(3)$

Selected bond length $(\mathrm{nm})$ and bond angles $\left({ }^{\circ}\right)$ : $\mathrm{Os}(1)-\mathrm{C}(1)=0.1734(4)$, $\mathrm{Os}(1)-\mathrm{H}(1)=0.1780(10), \mathrm{Os}(1)-\mathrm{P}(1)=0.23929(11), \mathrm{Os}(1)-\mathrm{P}(2)=$ $0.23885(11), \mathrm{Os}(1)-\mathrm{Cl}(1)=0.24799(9), \mathrm{Os}(1)-\mathrm{Cl}(2)=0.24583(11)$, $\mathrm{C}(1)-\mathrm{C}(2)=0.1422(5), \mathrm{C}(2)-\mathrm{C}(3)=0.1352(5), \mathrm{C}(3)-\mathrm{C}(4)=$ $0.1482(6), \mathrm{C}(3)-\mathrm{C}(5)=0.1473(6) ; \mathrm{Os}(1)-\mathrm{C}(1)-\mathrm{C}(2)=166.7(4)$, $\mathrm{C}(1)-\mathrm{C}(2)-\mathrm{C}(3)=125.5(4), \mathrm{P}(1)-\mathrm{Os}(1)-\mathrm{P}(2)=175.26(3), \mathrm{Cl}(1)-$ $\mathrm{Os}(1)-\mathrm{Cl}(2)=105.94(15), \mathrm{C}(1)-\mathrm{Os}(1)-\mathrm{Cl}(1)=163.73(15)$

化合物 3 的核磁数据与它的晶体结构一致, 其 ${ }^{31} \mathrm{P}\left\{{ }^{1} \mathrm{H}\right\}$ NMR 谱在 $\delta 6.0$ 处显示一个单峰信号: 在 ${ }^{1} \mathrm{H}$ $\mathrm{NMR}$ 谱中, $\mathrm{OsH}$ 质子信号位于 $\delta-6.37$ 处, 表现为三重 峰 $\left(J_{\mathrm{PH}}=16.0 \mathrm{~Hz}\right)$, 烯基质子信号位于 $\delta 3.74$ 处, 表现为
单峰. 在 ${ }^{13} \mathrm{C}\left\{{ }^{1} \mathrm{H}\right\}$ NMR 谱中, Os $\equiv \mathrm{C}$ 信号位于 $\delta 265.4$, 是个三重峰 $\left(J_{\mathrm{PC}}=11.9 \mathrm{~Hz}\right), \beta$-和 $\gamma$-位的烯基碳的信号分 别位于 $\delta 132.7$ 和 166.5 处.

$\mathrm{OsHCl}\left(\mathrm{PPh}_{3}\right)_{3}$ 与 3-氯-3-甲基-1-丁炔反应的可能机 理如 Scheme 2 所示. 16 电子的 $\mathrm{OsHCl}\left(\mathrm{PPh}_{3}\right)_{3}$ 与 3-氯-3甲基-1-丁炔反应可首先生成炔烃 $\pi$-配位中间体 $\mathbf{A}$ ，和 Grubbs 报道的钉氢配合物 $\mathrm{RuH}(\mathrm{H})_{2} \mathrm{Cl}\left(\mathrm{PCy}_{3}\right)_{2}$ 与炔丙基

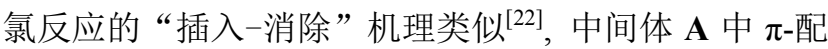
位的炔烃插入到 $\mathrm{Os}-\mathrm{H}$ 键生成锇的烯基配合物中间体 B，随后发生 $\gamma$-氯消除，同时消除的氯离子再配位到锇 中心得到五配位的锇烯基卡宾中间体 $\mathbf{C}$. 和钉体系的情 况不同的是，由于锇的还原性比钉强，在反应中更倾向 于生成配位饱和的含更多重金属一碳键的氧化异构体, 因此，中间体 $\mathbf{C}$ 不稳定，很容易发生 $\alpha-\mathrm{H}$ 消除而转化为 原子氢配位的烯基卡拜配合物 3. Werner 等 ${ }^{[25]}$ 也发现 $\mathrm{OsH}_{3} \mathrm{Cl}\left(\mathrm{PCy}_{3}\right)_{2}$ 与 3-氯-3-甲基-1-丁炔在低温下反应可生 成分子氢配位的锇烯基卡宾 $\mathrm{Os}\left(\mathrm{H}_{2}\right) \mathrm{Cl}_{2}(=\mathrm{CHCH}=$ $\left.\mathrm{CMe}_{2}\right)\left(\mathrm{PCy}_{3}\right)_{2}$, 但该化合物非常不稳定, 室温下易释放 出配位的氢分子而转化为锇一氢烯基卡拜 $\mathrm{OsHCl}_{2}($ 三 $\left.\mathrm{CCH}=\mathrm{CMe}_{2}\right)\left(\mathrm{PCy}_{3}\right)_{2}$. 与此类似, 我们 ${ }^{[5 \mathrm{f}]}$ 最近也发现锇 烯基卡拜 $\mathrm{OsCl}_{3}\left(\equiv \mathrm{CCH}=\mathrm{CPh}_{2}\right)\left(\mathrm{PCy}_{3}\right)_{2}$ 与 $\mathrm{NaOEt}$ 在室温 下反应可得到锇烯基卡宾 $\mathrm{OsCl}_{2}\left(=\mathrm{CCH}=\mathrm{CPh}_{2}\right)\left(\mathrm{PCy}_{3}\right)_{2}$, 但是该化合物也不稳定, 在溶液中易转化为热力学稳定 的钱一氢烯基卡拜 $\mathrm{OsHCl}_{2}\left(\equiv \mathrm{CCH}=\mathrm{CPh}_{2}\right)\left(\mathrm{PCy}_{3}\right)_{2}$.

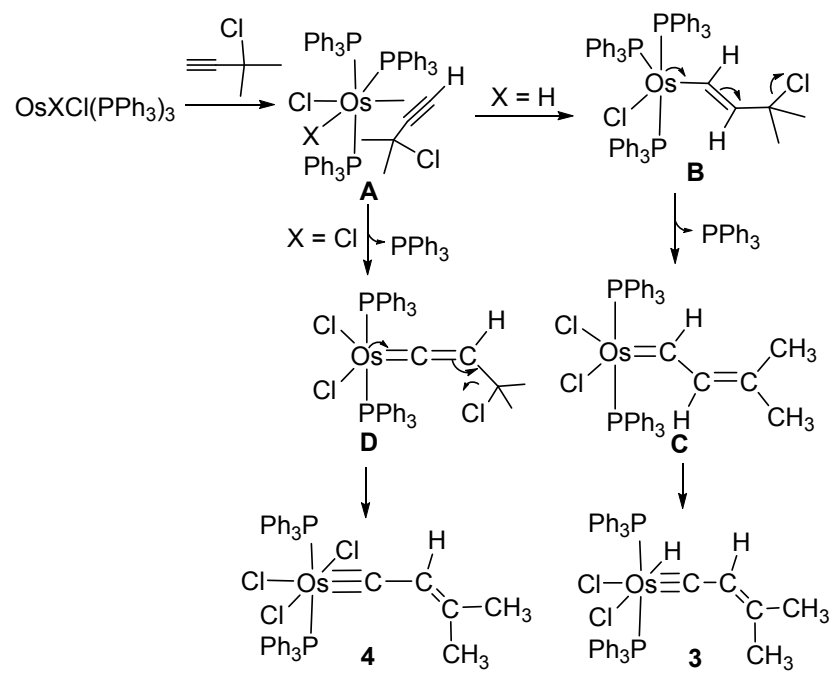

图式 $2 \mathrm{OsXCl}\left(\mathrm{PPh}_{3}\right)_{3}(\mathrm{X}=\mathrm{H}, \mathrm{Cl})$ 与 3-氯-3-甲基-1-丁炔反应 的可能机理

Scheme 2 Proposed mechanism for the reactions of OsXCl$\left(\mathrm{PPh}_{3}\right)_{3}(\mathrm{X}=\mathrm{H}, \mathrm{Cl})$ with 3-chloro-3-methylbut-1-yne

既然钉、锇的原子氢配合物可与炔丙基氯通过 “插 入-消除” 的机理进行反应, 作为平行研究, 我们也希望 探究不含原子氢配体的金属起始物与炔丙基氯反应的 情况，因此，考察了 $\mathrm{OsCl}_{2}\left(\mathrm{PPh}_{3}\right)_{3}$ 和 3-氯-3-甲基-1-丁炔 
的反应, 室温下两者物质的量比为 $1: 1.2$ 时反应缓慢, 但在 $100{ }^{\circ} \mathrm{C}$ 油浴下于甲苯中反应 $4 \mathrm{~h}$, 可析出大量粉红 色固体, 将反应液静置后, 通过 ${ }^{31} \mathrm{P}\left\{{ }^{1} \mathrm{H}\right\} \mathrm{NMR}$ 检测澄清 的溶液部分, 发现原料的信号消失, 除了在 $\delta-5.3$ 附 近有解离的 $\mathrm{PPh}_{3}$ 信号外, 在 $\delta-14.0$ 处有新的单峰信 号生成, 将上述反应液浓缩后, 过滤分离得到固体, 经 表征确认为三氯配位锇烯基卡拜配合物 $\mathrm{OsCl}_{3}$ (三 $\left.\mathrm{CCH}=\mathrm{CMe}_{2}\right)\left(\mathrm{PPh}_{3}\right)_{2}(4)$, 产率为 $77 \%$ (Scheme 1).

化合物 4 经过了 ${ }^{31} \mathrm{P}$ NMR, ${ }^{1} \mathrm{H}$ NMR 和 ${ }^{13} \mathrm{C}$ NMR 谱 及元素分析表征, 其 ${ }^{31} \mathrm{P}\left\{{ }^{1} \mathrm{H}\right\} \mathrm{NMR}$ 谱在 $\mathrm{CDCl}_{3}$ 中于 $\delta$ -14.1 处显示一个单峰信号, 在 ${ }^{1} \mathrm{H}$ NMR 谱中, 其烯基 质子信号在 $\delta 3.77$ 处, 表现为一个单峰, 在 ${ }^{13} \mathrm{C}\left\{{ }^{1} \mathrm{H}\right\}$ $\mathrm{NMR}$ 谱中, Os $\equiv C$ 信号为位于 $\delta 268.6$ 处的三重峰 $\left(J_{(\mathrm{PC})}=11.3 \mathrm{~Hz}\right), \beta$-和 $\gamma$-位的烯基碳的信号分别位于 $\delta$ 133.8 和 170.6 处, 与化合物 3 及已报道的三氯配位的 锇烯基卡拜配合物 $m e r-\mathrm{OsCl}_{3}\left(\equiv \mathrm{CCH}=\mathrm{CPh}_{2}\right)\left(\mathrm{PPh}_{3}\right)_{2}$ 相 应的 ${ }^{13} \mathrm{C}$ 信号接近 ${ }^{[5 \mathrm{p}]}$.

$\mathrm{OsCl}_{2}\left(\mathrm{PPh}_{3}\right)_{3}$ 与 3-氯-3-甲基-1-丁炔反应生成化合物 4 的可能机理如 Scheme 2 所示, 和 $\mathrm{OsHCl}\left(\mathrm{PPh}_{3}\right)_{3}$ 的反应 不同的是, 16 电子的 $\mathrm{OsCl}_{2}\left(\mathrm{PPh}_{3}\right)_{3}$ 与 3-氯-3-甲基-1-丁炔 发生 $\pi$-配位后, 中间体 $\mathbf{A}$ 中 $\pi$-配位的末端炔烃在锇上异 构化成亚乙基, 同时解离一个 $\mathrm{PPh}_{3}$ 配体, 生成中间体 $\mathbf{D}$, 中间体 $\mathbf{D}$ 可发生 $\gamma$-氯消除, 同时消除的氯离子再配 位到锇中心得到三氯配位的锇烯基卡拜 4. 端基炔在 $\mathrm{d}^{6}$ 电子八面体构型的配合物上发生表观的 1,2-氢迁移转化 成亚乙烯基配合物已是一个成熟反应 ${ }^{[18 \mathrm{a}, 28]}$, 而且已有 一系列报道表明, $\mathrm{OsCl}_{2}\left(\mathrm{PPh}_{3}\right)_{3}$ 可与不同的端基炔反应， 经过亚乙烯基中间体生成卡拜、亚丙二烯基、丙二烯基

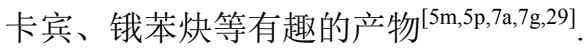

最近几年文献报道了许多与配合物 3 或 4 类似的 锇一氢烯基卡拜或三氯配位的锇烯基卡拜配合物, 特别 引人注目的是其中不少配合物的新奇反应 ${ }^{[4 a, 4 b, 7 a, 7 b, 8 a]}$. 例如, 锇-氢烯基卡拜 $\left[\mathrm{OsHCl}_{2}\left\{\equiv \mathrm{C}-\mathrm{C}\left(\mathrm{PPh}_{3}\right)=\mathrm{CHPh}\right\}-\right.$ $\left.\left(\mathrm{PPh}_{3}\right)_{2}\right]\left(\mathrm{BF}_{4}\right)$ 可以发生分子内的 $\mathrm{C}-\mathrm{H}$ 键活化反应生成 锇杂萘化合物 ${ }^{[8 \mathrm{e}]}$, 也可与乙腈发生 $[4+2]$ 环化反应得到 锇杂吡啶化合物 ${ }^{[8]}$; 三氯配位的锇烯基卡拜 $\left[\mathrm{OsCl}_{3}\{\equiv\right.$ $\left.\mathrm{CCH}=\mathrm{C}\left(2-\mathrm{ClC}_{6} \mathrm{H}_{4}\right)_{2}\right\}\left(\mathrm{PPh}_{3}\right)_{2}$ ] 在经 $\mathrm{Zn} / \mathrm{PPh}_{3}$ 还原可生成锇 杂䒺炔和 $\eta^{5}$-狮基配位的锇配合物 ${ }^{[7 \mathrm{e}]} ; \mathrm{OsCl}_{3}\{\equiv \mathrm{CCH}=$ $\left.\mathrm{C}\left(\mathrm{CMe}_{3}\right) \mathrm{CH}=\mathrm{CRCl}\right\}\left(\mathrm{PPh}_{3}\right)_{2}$ 用锌粉还原可以得到锇苯 炔化合物 $\left.\left[\mathrm{R}=\left(\mathrm{CH}_{2}\right)_{4} \mathrm{CH}_{3}\right)\right]$, 或锇苯炔发生还原消除而 生成锇卡宾产物 $(\mathrm{R}=\mathrm{H})^{[7 \mathrm{c}]}$, 这些锇一氢烯基卡拜配合物 主要通过锇的双原子氢配合物 $\mathrm{OsH}_{2} \mathrm{Cl}_{2}\left(\mathrm{PR}_{3}\right)_{2}(\mathrm{R}=\mathrm{Cy}$, $i$-Pr)与末端炔丙醇 $[5 \mathrm{f}, 13 \mathrm{c}, 18 \mathrm{a}]$ 、联 烯 ${ }^{[30]}$ 、共轭烯炔 ${ }^{[18 \mathrm{a}, \mathrm{c}]}$ 、 共轭二烯 ${ }^{[18 c]}$ 等有机底物反应制备 ${ }^{[4 a, 4 b]}$, 或是通过 $\mathrm{OsCl}_{2}\left(\mathrm{PPh}_{3}\right)_{3}$ 与炔丙醇反应后再与 $\mathrm{HBF}_{4}$ 作用得到 ${ }^{[8 \mathrm{f}]}$, 而
三氯配位的锇烯基卡拜配合物主要通过 $\mathrm{OsH}_{2} \mathrm{Cl}_{2}\left(\mathrm{PCy}_{3}\right)_{2}{ }^{[5 \mathrm{f}]}$ 或 $\mathrm{OsCl}_{2}\left(\mathrm{PPh}_{3}\right)_{3}{ }^{[5 \mathrm{~g}, 5 \mathrm{p}, 7 \mathrm{c}, 7 \mathrm{e}]}$ 与炔丙醇在 $\mathrm{HCl}$ 存在下一锅法反应制得. $\mathrm{OsXCl}\left(\mathrm{PPh}_{3}\right)_{3}(\mathrm{X}=\mathrm{H}, \mathrm{Cl})$ 与 3-氯-3-甲基-1-丁炔反应的结果表明，可望发展一个通 过简单易得的锇起始物 $\mathrm{OsHCl}\left(\mathrm{PPh}_{3}\right)_{3}$, 或 $\mathrm{OsCl}_{2}\left(\mathrm{PPh}_{3}\right)_{3}$ 与炔丙基氯反应合成这类锇一氢烯基卡拜和三氯配位的 锇烯基卡拜配合物的简便方法.

\section{$1.2 \mathrm{OsXCl}_{2}\left(\equiv \mathrm{CCH}=\mathrm{CMe}_{2}\right)\left(\mathrm{PPh}_{3}\right)_{2}(\mathrm{X}=\mathrm{H}, \mathrm{Cl})$ 与二 苯基(苯乙炔基)膦的反应}

从烯烃复分解反应的经验可以看出, 膦配体对钉卡 宾配合物 $\mathrm{RuCl}_{2}(=\mathrm{CHPh})\left(\mathrm{PR}_{3}\right)_{2}$ 在烯烃复分解反应中的 活性起着至关重要的作用 ${ }^{[20 \mathrm{c}]}$ ，二苯基(苯乙炔基)膦 $\left(\mathrm{Ph}_{2} \mathrm{PC} \equiv \mathrm{CPh}\right)$ 与 $\mathrm{PPh}_{3} 、 \mathrm{PCy} 、 \mathrm{P}(i-\mathrm{Pr})_{3}$ 等膦配体相比，具 有体积小、给电子能力相对较弱的特点, 而且目前还未 见炔基膦配位的锇卡拜配合物的报道，因此，我们也考 察了卡拜配合物 $\mathrm{OsXCl}_{2}\left(\equiv \mathrm{CCH}=\mathrm{CMe}_{2}\right)\left(\mathrm{PPh}_{3}\right)_{2}(\mathrm{X}=\mathrm{H}$, 3; $\mathrm{X}=\mathrm{Cl}, 4)$ 与二苯基(苯乙炔基)膦的反应. 室温下，锇 氢卡拜化合物 3 与 $\mathrm{Ph}_{2} \mathrm{PC} \equiv \mathrm{CPh}$ 配体在二氯甲烷中反应 非常缓慢，升高至 $40{ }^{\circ} \mathrm{C}$ 反应时，发现 $8 \mathrm{~h}$ 后化合物 3 还 未能反应完全, 而当化合物 3 与 $\mathrm{Ph}_{2} \mathrm{PC} \equiv \mathrm{CPh}$ (物质的量 比为 $1: 5$ ) 在二氯甲烷中 $50{ }^{\circ} \mathrm{C}$ 下于耐压管中反应, $4 \mathrm{~h}$ 即可完全转化为 3 个 $\mathrm{Ph}_{2} \mathrm{PC} \equiv \mathrm{CPh}$ 配位的锇烯基卡宾配 合物 $\mathrm{OsCl}_{2}\left(=\mathrm{CHCH}=\mathrm{CMe}_{2}\right)\left(\mathrm{Ph}_{2} \mathrm{PC} \equiv \mathrm{CPh}\right)_{3}(\mathbf{5})$, 分离产 率为 $86 \%$ (Scheme 3).

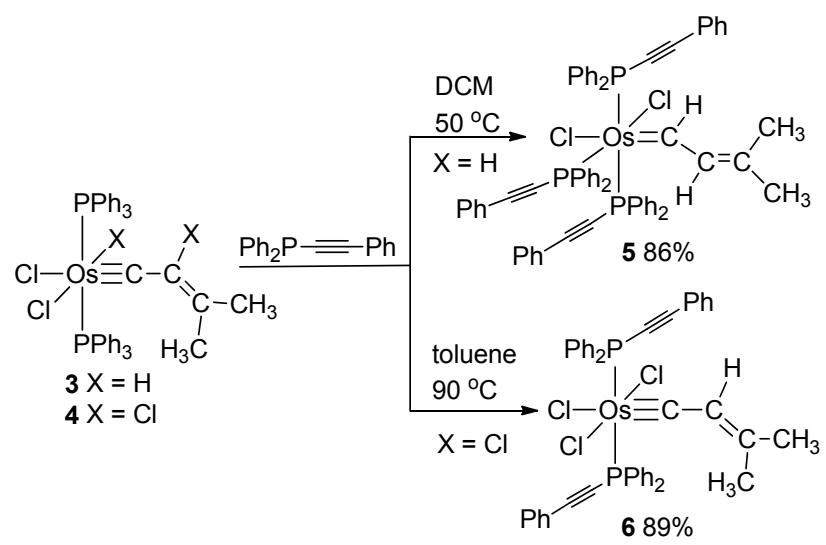

图式 $3 \mathrm{OsXCl} 2\left(\equiv \mathrm{CCH}=\mathrm{CMe}_{2}\right)\left(\mathrm{PPh}_{3}\right)_{2}(\mathrm{X}=\mathrm{H}, \mathrm{Cl})$ 与二苯基 (苯乙炔基)膦的反应

Scheme 3 Reactions of $\mathrm{OsXCl}_{2}\left(\equiv \mathrm{CCH}=\mathrm{CMe}_{2}\right)\left(\mathrm{PPh}_{3}\right)_{2}(\mathrm{X}=$ $\mathrm{H}, \mathrm{Cl}$ ) with $\mathrm{Ph}_{2} \mathrm{PC} \equiv \mathrm{CPh}$

化合物 5 经过了 ${ }^{31} \mathrm{P}$ NMR, ${ }^{1} \mathrm{H}$ NMR 和 ${ }^{13} \mathrm{C}$ NMR 谱 及元素分析表征. 其 ${ }^{31} \mathrm{P}\left\{{ }^{1} \mathrm{H}\right\} \mathrm{NMR}$ 谱在 $\mathrm{CDCl}_{3}$ 中表现为 一对未完全䢃裂开的三重峰和两重峰, 化学位移分别为 $\delta-28.1$ 和 -28.6 , 对应积分比例为 $1: 2$, 表明存在两 种不等价的膦配体, 分别对应赤道面的一个膦和轴向处 于对位的两个膦配体的信号. 在 ${ }^{1} \mathrm{H}$ NMR 谱中, 卡宾氢 
的信号位于 $\delta 21.75$, 显示为一个宽峰, 但可由 ${ }^{1} \mathrm{H}\left\{{ }^{31} \mathrm{P}\right\}$ 确认其受邻位烯基质子的偶合而䢃裂成两重峰 $\left(J_{\mathrm{HH}}=\right.$ $12.0 \mathrm{~Hz}$ ), 其烯基质子信号在 $\delta 6.98$ 处, 表现为一个两重 峰 $\left(J_{\mathrm{HH}}=12.0 \mathrm{~Hz}\right.$, 与苯基质子信号有部分重叠). 在 ${ }^{13} \mathrm{C}\left\{{ }^{1} \mathrm{H}\right\}$ NMR 谱中, 化合物 $\mathbf{5}$ 的 $\mathrm{Os}=\mathrm{C}$ 信号位于 $\delta 274.9$ 处, 由于受到 3 个膦配体的偶合, 表现为多重峰, $\beta$-和 $\gamma$ 位的烯基碳的信号分别位于 $\delta 157.2$ 和 145.7 处. 特别是 $\delta 84.3\left(\mathrm{~d}, J_{\mathrm{PC}}=83.4 \mathrm{~Hz}\right)$ 和 $83.0\left(\mathrm{t}, J_{\mathrm{PC}}=47.9 \mathrm{~Hz}\right)$ 处显示 两种炔基膦 $\mathrm{Ph}_{2} \mathrm{PC}_{\alpha} \equiv \mathrm{CPh}$ 的炔基 $\mathrm{C}_{\alpha}$ 信号, 很清楚地反 映出化合物 5 中的 3 个膦配体都为炔基膦配体, 前者为 两重峰, 表明只受一个磷的偶合, 从偶合常数可以看出 为炔基的 $\mathrm{C}_{\alpha}$ 信号, 因此可清楚地归属为赤道面上的一 个炔基膦上炔基的 $\mathrm{C}_{\alpha}$ 信号; 后者为轴向的两个膦配体 的炔基 $\mathrm{C}_{\alpha}$ 信号, 因处于对位的这两个膦配体对其的虚 拟偶合(Virtual Coupling)而表现为三重峰. 与此相一致, 对应的两种炔基膦 $\mathrm{Ph}_{2} \mathrm{PC} \equiv \mathrm{C}_{\beta} \mathrm{Ph}$ 的炔基 $\mathrm{C}_{\beta}$ 信号分别位 于 $\delta 109.73\left(\mathrm{~d}, J_{\mathrm{PC}}=8.34 \mathrm{~Hz}\right.$, 赤道面 $)$ 和 $109.50\left(\mathrm{t}, J_{\mathrm{PC}}=\right.$ $6.1 \mathrm{~Hz}$, 轴向).

锇-氢卡拜化合物 3 与 $\mathrm{Ph}_{2} \mathrm{PC} \equiv \mathrm{CPh}$ 反应生成化合 物 5 的可能机理如 Scheme 4 所示. 锇-氢烯基卡拜配合 物 3 首先与 $\mathrm{Ph}_{2} \mathrm{PC} \equiv \mathrm{CPh}$ 发生配体取代反应, 生成双 $\mathrm{Ph}_{2} \mathrm{PC} \equiv \mathrm{CPh}$ 配位的锇-氢烯基卡拜配合物 $\mathbf{A}$, 由于 $\mathrm{Ph}_{2} \mathrm{PC} \equiv \mathrm{CPh}$ 的给电子能力较 $\mathrm{PPh}_{3}$ 弱, 因此, $\mathrm{Ph}_{2} \mathrm{PC} \equiv$ $\mathrm{CPh}$ 的配位降低了锇中心的电子密度, 相应削弱了 $\mathrm{Os}-\mathrm{H}$ 及 $\mathrm{Os} \equiv \mathrm{C}$ 键的强度, 从而也降低了 $\mathrm{H}$ 配体从锇 迁移到卡拜 $\alpha$-碳上的能垒, 使得锇-氢烯基卡拜 $\mathbf{A}$ 和锇 烯基卡宾中间体 $\mathbf{B}$ 之间的平衡有利于移向后者, 并且由 于 $\mathrm{Ph}_{2} \mathrm{PC} \equiv \mathrm{CPh}$ 的空间位阻较小, 16 电子的中间体 $\mathbf{B}$ 可 进一步与另一个 $\mathrm{Ph}_{2} \mathrm{PC} \equiv \mathrm{CPh}$ 配位, 生成热力学更稳定 的 18 电子产物 $\mathbf{5}$.
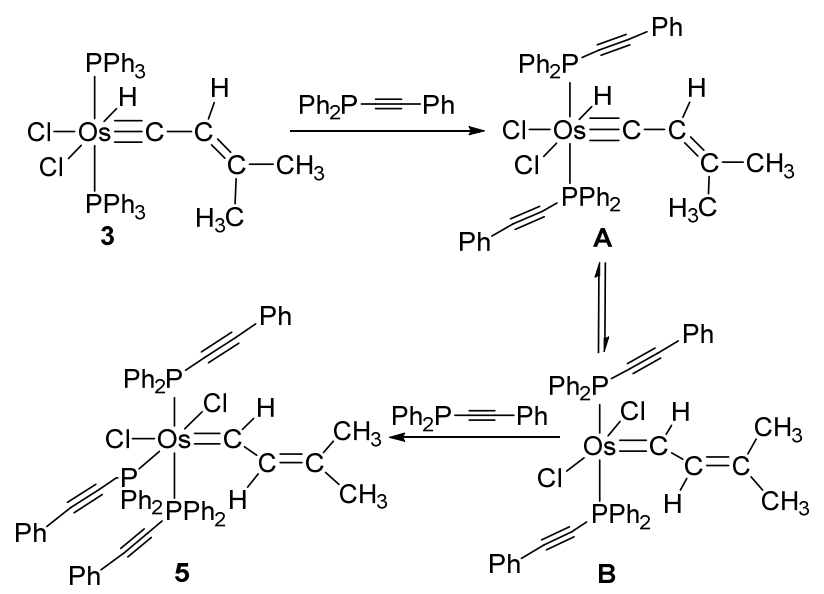

图式 $4 \mathrm{OsHCl}_{2}\left(\equiv \mathrm{CCH}=\mathrm{CMe}_{2}\right)\left(\mathrm{PPh}_{3}\right)_{2}$ 与二苯基(苯乙炔基) 膦反应的可能机理

Scheme 4 Proposed mechanism for the reaction of $\mathrm{OsHCl}_{2}(\equiv$ $\left.\mathrm{CCH}=\mathrm{CMe}_{2}\right)\left(\mathrm{PPh}_{3}\right)_{2}$ with $\mathrm{Ph}_{2} \mathrm{PC} \equiv \mathrm{CPh}$
锇处于第三过渡系, 在锇-氢卡拜 $\mathrm{L}_{n} \mathrm{OsH}(\equiv \mathrm{CR})$ 与 锇卡宾 $\mathrm{L}_{n} \mathrm{Os}(=\mathrm{CHR})$ 这对氧化-还原异构体的平衡中, 通常情况下以其氧化异构体 $\mathrm{L}_{n} \mathrm{OsH}(\equiv \mathrm{CR})$ 更稳定. DFT

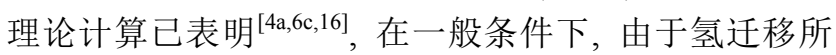
需要的能量较高, 所以不易发生锇上的氢配体到卡拜 $\alpha$ 碳上的迁移，但是氢迁移的能垒与金属中心的电子富足 程度有关, 因此可以通过改变配合物中的辅助配体的电 子性质来调控金属中心的电子密度，从而控制两者之间 的衡. 例如, Caulton 小组 ${ }^{[31]}$ 发现 $\pi$ 酸配体 $\mathrm{CO}$ 能有效地 促进 $\mathrm{OsHCl}_{2}\left(\equiv \mathrm{CCH}_{2} \mathrm{R}\right)\left\{\mathrm{P}(i-\mathrm{Pr})_{3}\right\}_{2}(\mathrm{R}=\mathrm{Ph}, \mathrm{Me})$ 转化为卡 宾配合物 $\mathrm{OsCl}_{2}\left(=\mathrm{CHCH}_{2} \mathrm{R}\right)(\mathrm{CO})\left\{\mathrm{P}(i-\mathrm{Pr})_{3}\right\}_{2}$. Esteruelas 小组 ${ }^{[6 c]}$ 通过实验与理论计算相结合, 发现依次将锇-氢 卡拜 $\mathrm{OsHCl}_{2}\left(\equiv \mathrm{CCH}=\mathrm{CPh}_{2}\right)\left\{\mathrm{P}(i-\mathrm{Pr})_{3}\right\}_{2}$ 中的 $\mathrm{Cl}$ 配体取代 为乙腈配体, 使其依次转化为离子型卡拜配合物 $\left[\mathrm{OsHCl}\left(\equiv \mathrm{CCH}=\mathrm{CPh}_{2}\right)(\mathrm{MeCN})\left\{\mathrm{P}(i-\mathrm{Pr})_{3}\right\}_{2}\right]\left(\mathrm{BF}_{4}\right)$ 和 $\left[\mathrm{OsH}\left(\equiv \mathrm{CCH}=\mathrm{CPh}_{2}\right)(\mathrm{MeCN})_{2}\left\{\mathrm{P}(i-\mathrm{Pr})_{3}\right\}_{2}\right]\left(\mathrm{BF}_{4}\right)_{2}$ 后，可逐 步降低金属中心的电子密度, 从而也逐步降低了金属中 心到卡拜 $\alpha$-碳上氢迁移的能垒, 使得其越来越容易转化 为相应的五配位卡宾物种, 并在溶液中易与过量的乙腈 进一步配位得到更稳定的卡宾产物 $[\mathrm{OsCl}(=\mathrm{CHCH}=$ $\left.\mathrm{CPh}_{2}\right)(\mathrm{MeCN})_{2}\left\{\mathrm{P}\left(i-\mathrm{Pr}_{3}\right\}_{2}\right]\left(\mathrm{BF}_{4}\right)$ 和 $[\mathrm{Os}(=\mathrm{CHCH}=$ $\left.\left.\mathrm{CPh}_{2}\right)(\mathrm{MeCN})_{3}\left\{\mathrm{P}(i-\mathrm{Pr})_{3}\right\}_{2}\right]\left(\mathrm{BF}_{4}\right)_{2}$. 在上述反应中, 通过 $\mathrm{Ph}_{2} \mathrm{PC} \equiv \mathrm{CPh}$ 对锇-氢卡拜 3 中的 $\mathrm{PPh}_{3}$ 配体的取代, 实现 了锇一氢卡拜转化为卡宾的调控.

我们也考察了 $\mathrm{OsCl}_{3}\left(\equiv \mathrm{CCH}=\mathrm{CMe}_{2}\right)\left(\mathrm{PPh}_{3}\right)_{2}$ (4) 与 $\mathrm{Ph}_{2} \mathrm{PC} \equiv \mathrm{CPh}$ 的反应, 化合物 $\mathbf{4}$ 与 $\mathrm{Ph}_{2} \mathrm{PC} \equiv \mathrm{CPh}$ 混合物的 物质的量比为 $1: 5$ 时, 在甲苯中于 $90{ }^{\circ} \mathrm{C}$ 下反应, 并在 反应过程中通过检测反应液的 ${ }^{31} \mathrm{P}\left\{{ }^{1} \mathrm{H}\right\}$ NMR 跟踪反应 情况, 发现 $5 \mathrm{~h}$ 后反应尚未完全且变得非常缓慢, 这时 ${ }^{31} \mathrm{P}\left\{{ }^{1} \mathrm{H}\right\}$ NMR 谱显示有 4 种含膦化合物的信号, 即除了 过量的 $\mathrm{Ph}_{2} \mathrm{PC} \equiv \mathrm{CPh}$ 配体的信号和解离的 $\mathrm{PPh}_{3}$ 的信号、 以及化学位移位于 $\delta-28.0$ 附近的新生成产物的信号 外，还有部分未反应完全的原料 $\mathbf{4}$ 的膦信号(约 30\%), 显然, 由于溶液中解离的 $\mathrm{PPh}_{3}$ 和 $\mathrm{Ph}_{2} \mathrm{PC} \equiv \mathrm{CPh}$ 配体存在 着竞争, 使得反应达到一个平衡而未能进行完全, 因此, 需要先分离除去体系中游离的 $\mathrm{PPh}_{3}$ 后, 所得固体产物 再次与过量的 $\mathrm{Ph}_{2} \mathrm{PC} \equiv \mathrm{CPh}$ 反应继续在甲苯中于 $90{ }^{\circ} \mathrm{C}$ 下加热反应 $5 \mathrm{~h}$ 才可使得反应进行完全，经分离以 $89 \%$ 的产率得到纯的二苯基(苯乙炔基)膦双取代的锇烯基卡 拜化合物 $\mathrm{OsCl}_{3}\left(\equiv \mathrm{CCH}=\mathrm{CMe}_{2}\right)\left(\mathrm{Ph}_{2} \mathrm{PC} \equiv \mathrm{CPh}\right)_{2} \quad$ (6) (Scheme 3).

化合物 6 经过了 ${ }^{31} \mathrm{P}$ NMR, ${ }^{1} \mathrm{H}$ NMR 和 ${ }^{13} \mathrm{C}$ NMR 谱 及元素分析表征进行了核磁表征. 其 ${ }^{31} \mathrm{P}\left\{{ }^{1} \mathrm{H}\right\} \mathrm{NMR}$ 谱在 $\mathrm{CDCl}_{3}$ 中显示 $\delta-28.2$ 处的一个单峰信号, 在 ${ }^{1} \mathrm{H}$ NMR 谱中, 其烯基质子信号在 $\delta 4.21$ 处, 表现为一个单峰. 
在 ${ }^{13} \mathrm{C}\left\{{ }^{1} \mathrm{H}\right\}$ NMR 谱中，化合物 6 的 Os $\equiv \mathrm{C}$ 信号位于 $\delta$ 269.1 处, 表现为三重峰 $\left(J_{\mathrm{PC}}=11.3 \mathrm{~Hz}\right), \beta$-和 $\gamma$-位的烯基 碳的信号分别位于 $\delta 173.8$ 和 121.3 处, $\mathrm{Ph}_{2} \mathrm{PC}_{\alpha} \equiv \mathrm{C}_{\beta} \mathrm{Ph}$ 配 体的两个炔基碳信号分别位于 $\delta 112.7$ 处和 79.3 处, 分 别对应炔基的 $\mathrm{C}_{\beta}$ 和 $\mathrm{C}_{\alpha}$ 的信号, 因处于对位的这两个膦 配体对其的虚拟偶合(Virtual Coupling), 各自都被䢃裂 为三重峰, 偶合常数分别为二重峰 $J_{\mathrm{PC}}=8.6 \mathrm{~Hz}$ 和 $J_{\mathrm{PC}}=$ $54.2 \mathrm{~Hz}$.

\section{2 结论}

研究了 $\mathrm{OsHCl}\left(\mathrm{PPh}_{3}\right)_{3}$ 和 $\mathrm{OsCl}_{2}\left(\mathrm{PPh}_{3}\right)_{3}$ 分别与 3-氯-3甲基-1-丁炔反应, 简便地合成了锇-氢烯基卡拜配合物 $\mathrm{OsHCl}_{2}\left(\equiv \mathrm{CCH}=\mathrm{CMe}_{2}\right)\left(\mathrm{PPh}_{3}\right)_{2}(3)$ 和三氯配位的锇烯基 卡拜配合物 $\mathrm{OsCl}_{3}\left(\equiv \mathrm{CCH}=\mathrm{CMe}_{2}\right)\left(\mathrm{PPh}_{3}\right)_{2}$ (4), 前者的反 应经炔烃插入及 $\gamma-\mathrm{Cl}$ 消除产生五配位的烯基卡宾中间 体, 再发生 $\alpha-\mathrm{H}$ 消除转化成 3, 后者的反应经亚乙烯基 中间体的 $\gamma-\mathrm{Cl}$ 消除得到 $\mathbf{4}$, 因此, 可望将此炔丙基氯的 $\gamma-\mathrm{Cl}$ 消除反应拓展为一个由简单易得的 $\mathrm{OsXCl}\left(\mathrm{PPh}_{3}\right)_{3}$ $(\mathrm{X}=\mathrm{H}, \mathrm{Cl})$ 起始物合成这类锇-氢烯基卡拜和三氯配位 的锇烯基卡拜配合物的简便方法. 化合物 3 与二苯基 (苯乙炔基)膦反应可转化为 3 个二苯基(苯乙炔基)膦配 位的锇烯基卡宾配合物 $\mathrm{OsCl}_{2}\left(=\mathrm{CHCH}=\mathrm{CMe}_{2}\right)$ $\left(\mathrm{Ph}_{2} \mathrm{PC} \equiv \mathrm{CPh}\right)_{3}(\mathbf{5})$, 而化合物 4 与二苯基(苯乙炔基)膦 反应得到膦配体取代产物 $\mathrm{OsCl}_{3}\left(\equiv \mathrm{CCH}=\mathrm{CMe}_{2}\right)$ $\left(\mathrm{Ph}_{2} \mathrm{PC} \equiv \mathrm{CPh}\right)_{2}$ (6). 二苯基(苯乙炔基)膦配体对 锇-氢 卡拜 3 中的 $\mathrm{PPh}_{3}$ 配体取代后, 因其较弱的供电子能力降 低了锇中心的电子密度, 促成了锇上的原子氢配体到卡 拜 $\alpha$-碳的 1,2-迁移而转化为卡宾物种, 同时因二苯基(苯 乙炔基)膦配体较小的空间位阻可进一步与锇中心配位, 因此生成了更稳定的 18 电子的六配位卡宾配合物 $\mathbf{5}$, 实 现了膦配体调控的锇-氢卡拜向卡宾配合物的转化.

\section{3 实验部分}

除非特别说明, 所有实验均采用标准的 Schlenk 实 验技术，在氩气保护的无水无氧条件下进行.

\section{1 仪器与试剂}

所有溶剂均为分析纯级, 四氢呋喃(THF)、乙醚、正 己烷、甲苯使用前均在氩气氛中经钠-二苯甲酮回流处 理, 二氯甲烷使用前经氢化钙回流处理, OsHCl$\left(\mathrm{PPh}_{3}\right)_{3}{ }^{[26]} 、 \mathrm{OsCl}_{2}\left(\mathrm{PPh}_{3}\right)_{3}{ }^{[32]} 、 \mathrm{Ph}_{2} \mathrm{PC} \equiv \mathrm{CPh}^{[33]}$ 根据文献方 法合成, 其他试剂均从 Sigma-Aldrich, ACROS 和 Alfa-Aesar 购买.

测试仪器：核磁共振谱 ${ }^{1} \mathrm{H} N \mathrm{NMR} 、{ }^{31} \mathrm{P}\left\{{ }^{1} \mathrm{H}\right\} \mathrm{NMR}$ 、 ${ }^{13} \mathrm{C}\left\{{ }^{1} \mathrm{H}\right\}$ NMR 在 Bruker AV400 (400 MHz)或 Bruker AV500 (500 MHz)核磁共振仪上测定, ${ }^{1} \mathrm{H} N \mathrm{NMR} 、{ }^{13} \mathrm{C}\left\{{ }^{1} \mathrm{H}\right\}$
NMR 采用 TMS 定标; ${ }^{31} \mathrm{P}\left\{{ }^{1} \mathrm{H}\right\} \mathrm{NMR}$ 采用 $85 \% \mathrm{H}_{3} \mathrm{PO}_{4}$ 定 标, 元素分析使用 Carlo Erba Instruments 仪器公司生产 的 Vario EL 型元素分析仪, 熔点用 Yanaco MP-500 型显 微熔点仪(温度计未校正)测定, 如无特别说明, 操作温 度为 $298 \mathrm{~K}$.

\section{2 实验步骤}

\subsection{1 $\mathrm{OsHCl}_{2}\left(\equiv \mathrm{CCH}=\mathrm{CMe}_{2}\right)\left(\mathrm{PPh}_{3}\right)_{2}(3)$ 的合成}

往 $\mathrm{OsHCl}\left(\mathrm{PPh}_{3}\right)_{3}(400 \mathrm{mg}, 0.39 \mathrm{mmol})$ 的甲苯 $(15$ $\mathrm{mL}$ )悬浊液中加入 3 -氯-3-甲基-1-丁炔 $(53 \mu \mathrm{L}, 0.47$ $\mathrm{mmol}$ ), 室温下搅拌反应 $4 \mathrm{~h}$, 生成大量棕色沉淀, 将反 应液浓缩至约 $5 \mathrm{~mL}$, 过滤得到固体, 经乙醚 $(5 \mathrm{~mL} \times 2)$ 洗涤后，真空下抽干，收集得到棕色固体 $265 \mathrm{mg}$, 产率 $80 \%$. m.p. $220 \sim 229{ }^{\circ} \mathrm{C}$ (分解); ${ }^{1} \mathrm{H}$ NMR $\left(\mathrm{CDCl}_{3}, 400\right.$ MHz) $\delta$ : $-6.37\left(\mathrm{t}, J_{\mathrm{PH}}=16.0 \mathrm{~Hz}, 1 \mathrm{H}, \mathrm{OsH}\right), 1.00(\mathrm{~s}, 3 \mathrm{H}$, $\left.\mathrm{CH}_{3}\right), 1.17\left(\mathrm{~s}, 3 \mathrm{H}, \mathrm{CH}_{3}\right), 3.74(\mathrm{~s}, 1 \mathrm{H}, \mathrm{CH}=\mathrm{C}), 7.26 \sim 7.37$ $(\mathrm{m}, 18 \mathrm{H}, \mathrm{Ph}), 7.84 \sim 7.86(\mathrm{~m}, 12 \mathrm{H}, \mathrm{Ph}) ;{ }^{13} \mathrm{C}\left\{{ }^{1} \mathrm{H}\right\}$ NMR $\left(\mathrm{CDCl}_{3}, 101 \mathrm{MHz}\right) \delta: 265.4\left(\mathrm{t}, J_{(\mathrm{PC})}=11.9 \mathrm{~Hz}, \mathrm{Os} \equiv \mathrm{C}\right)$, $166.5\left[\mathrm{~s},=\mathbf{C}\left(\mathrm{CH}_{3}\right)_{2}\right], 134.9\left(\mathrm{t}, J_{(\mathrm{PC})}=5.1 \mathrm{~Hz}\right.$, ortho $\left.-\mathrm{PPh}_{3}\right)$, $133.3\left(\mathrm{t}, J_{\mathrm{PC}}=26.4 \mathrm{~Hz}\right.$, ipso- $\left.\mathrm{PPh}_{3}\right), 132.7$ (s, CH=), 130.0 $\left(\mathrm{s}\right.$, para $\left.-\mathrm{PPh}_{3}\right), 127.8\left(\mathrm{t}, J_{\mathrm{PC}}=5.2 \mathrm{~Hz}\right.$, meta $\left.-\mathrm{PPh}_{3}\right), 26.4(\mathrm{~s}$, $\left.\mathrm{CH}_{3}\right), 21.9\left(\mathrm{~s}, \mathrm{CH}_{3}\right) ;{ }^{31} \mathrm{P}\left\{{ }^{1} \mathrm{H}\right\}$ NMR $\left(\mathrm{CDCl}_{3}, 162 \mathrm{MHz}\right) \delta$ : 6.0. Anal. calcd for $\mathrm{C}_{41} \mathrm{H}_{38} \mathrm{Cl}_{2} \mathrm{OsP}_{2}$ : $\mathrm{C} 57.67, \mathrm{H}$ 4.49; found C 57.98, H 4.86 .

将正已烷溶剂缓慢注入到该化合物的二氯甲烷溶 液的上层，通过缓慢扩散得到棕红色块状晶体.

\subsection{2 $\mathrm{OsCl}_{3}\left(\equiv \mathrm{CCH}=\mathrm{CMe}_{2}\right)\left(\mathrm{PPh}_{3}\right)_{2}$ (4) 的合成}

往 $\mathrm{OsCl}_{2}\left(\mathrm{PPh}_{3}\right)_{3}(400 \mathrm{mg}, 0.38 \mathrm{mmol})$ 的甲苯 $(20$ $\mathrm{mL}$ )溶液中加入 3 -氯-3-甲基-1-丁炔 $(52 \mu \mathrm{L}, 0.46 \mathrm{mmol})$, 反应液在 $100{ }^{\circ} \mathrm{C}$ 下加热搅拌反应 $4 \mathrm{~h}$, 生成大量粉红色 沉淀，过滤得到固体，经乙醚 $(10 \mathrm{~mL} \times 2)$ 洗涤后，真空 下抽干，收集得到粉色固体 $198 \mathrm{mg}$; 滤液减压浓缩至2 $\mathrm{mL}$, 加入乙醚 $(20 \mathrm{~mL})$ 搅拌, 生成粉色沉淀, 经乙醚 $(10$ $\mathrm{mL} \times 2)$ 洗涤后，真空下抽干，又收集得到部分粉色固体 $62 \mathrm{mg}$, 总产量 $260 \mathrm{mg}$, 产率 $77 \%$. m.p. $235 \sim 244{ }^{\circ} \mathrm{C}$ (分 解); ${ }^{1} \mathrm{H}$ NMR $\left(\mathrm{CDCl}_{3}, 500 \mathrm{MHz}\right) \delta: 1.09$ (s, 3H, $\left.\mathrm{CH}_{3}\right), 1.39$ (s, $\left.3 \mathrm{H}, \mathrm{CH}_{3}\right), 3.77(\mathrm{~s}, 1 \mathrm{H}, \mathbf{C H}=\mathrm{C}), 7.34 \sim 7.36(\mathrm{~m}, 18 \mathrm{H}$, $\left.\mathrm{PPh}_{3}\right), 7.94 \sim 7.95\left(\mathrm{~m}, 12 \mathrm{H}, \mathrm{PPh}_{3}\right),{ }^{13} \mathrm{C}\left\{{ }^{1} \mathrm{H}\right\} \mathrm{NMR}\left(\mathrm{CDCl}_{3}\right.$, $126 \mathrm{MHz}) \delta: 268.6\left(\mathrm{t}, J_{\mathrm{PC}}=11.3 \mathrm{~Hz}, \mathrm{Os} \equiv \mathrm{C}\right), 170.6[\mathrm{~s},=$ $\left.\mathrm{C}\left(\mathrm{CH}_{3}\right)_{2}\right], 135.2\left(\mathrm{t}, J_{\mathrm{PC}}=4.4 \mathrm{~Hz}\right.$, ortho $\left.-\mathrm{PPh}_{3}\right), 133.8(\mathrm{~s}$, $\mathrm{CH}=), 131.0\left(\mathrm{t}, J_{\mathrm{PC}}=26.2 \mathrm{~Hz}\right.$, ipso- $\left.\mathrm{PPh}_{3}\right), 130.0(\mathrm{~s}$, para- $\left.\mathrm{PPh}_{3}\right), 127.5\left(\mathrm{t}, J_{\mathrm{PC}}=4.6 \mathrm{~Hz}\right.$, meta $\left.-\mathrm{PPh}_{3}\right), 26.6(\mathrm{~s}$, $\left.\mathrm{CH}_{3}\right), 24.5\left(\mathrm{~s}, \mathrm{CH}_{3}\right) ;{ }^{31} \mathrm{P}\left\{{ }^{1} \mathrm{H}\right\}$ NMR $\left(\mathrm{CDCl}_{3}, 202 \mathrm{MHz}\right) \delta$ : -14.1. Anal. calcd for $\mathrm{C}_{41} \mathrm{H}_{37} \mathrm{Cl}_{3} \mathrm{OsP}_{2}$ : C 55.44, H 4.20; found C 55.86, H 4.54. 
3.2.3 $\mathrm{OsCl}_{2}\left(=\mathrm{CHCH}=\mathrm{CMe}_{2}\right)\left(\mathrm{Ph}_{2} \mathrm{PC} \equiv \mathrm{CPh}\right)_{3}(5)$ 的 合成

将 $\mathrm{OsHCl}_{2}\left(\equiv \mathrm{CCH}=\mathrm{CMe}_{2}\right)\left(\mathrm{PPh}_{3}\right)_{2}$ (3) (150 mg, 0.18 $\mathrm{mmol})$ 和 $\mathrm{Ph}_{2} \mathrm{PC} \equiv \mathrm{CPh}(252 \mathrm{mg}, 0.88 \mathrm{mmol})$ 的混合物在 耐压反应管中溶于二氯甲烷 $(6 \mathrm{~mL})$ 中, 在 $50{ }^{\circ} \mathrm{C}$ 浴温下 加热搅拌反应 $4 \mathrm{~h}$, 溶液颜色由黄色变为浅绿色, 将反 应液转移入 $50 \mathrm{~mL}$ 的 Schlenk 瓶中, 在减压条件下浓缩 至 $2 \mathrm{~mL}$, 并在持续搅拌下加入乙醚 $(20 \mathrm{~mL})$, 生成浅绿 色沉淀, 过滤得到固体, 经乙醚 $(5 \mathrm{~mL} \times 2)$ 洗涤后, 真 空下抽干, 收集得到浅绿色固体 $180 \mathrm{mg}$, 产率 $86 \%$. m.p. $288 \sim 298{ }^{\circ} \mathrm{C}$ (分解); ${ }^{1} \mathrm{H} \mathrm{NMR}\left(\mathrm{CDCl}_{3}, 400 \mathrm{MHz}\right) \delta$ : $21.75\left(\mathrm{br}, J_{\mathrm{HH}}=12.0 \mathrm{~Hz}, 1 \mathrm{H}, \mathrm{Os}=\mathrm{CH}\right), 8.10 \sim 6.75[\mathrm{~m}, 46$ $\mathrm{H}, \mathrm{Ph}$ and $\mathrm{CH}=$, 其中烯基质子信号位于 $6.98\left(\mathrm{~d}, J_{\mathrm{HH}}=\right.$ $12.0 \mathrm{~Hz}$, 经 $\mathrm{H}-\mathrm{H} \mathrm{COSY}$ 确认), 与苯基质子信号有部分 重叠], $1.28\left(\mathrm{~s}, 3 \mathrm{H}, \mathrm{CH}_{3}\right), 0.51\left(\mathrm{~s}, 3 \mathrm{H}, \mathrm{CH}_{3}\right) ;{ }^{13} \mathrm{C}\left\{{ }^{1} \mathrm{H}\right\} \mathrm{NMR}$ $\left(\mathrm{CDCl}_{3}, 101 \mathrm{MHz},\right) \delta: 274.9(\mathrm{~m}, \mathrm{Os}=\mathrm{CH}), 157.2(\mathrm{~s}$, $\left.\mathrm{CH}=\mathrm{CMe}_{2}\right), 145.7\left(\mathrm{~s},=\mathrm{CMe}_{2}\right), 138.0\left(\mathrm{~d}, J_{\mathrm{PC}}=60.8 \mathrm{~Hz}\right.$, ipso- $\mathrm{PPh}_{2}$, trans to $\mathrm{Cl}$ ), 134.3 (t, $J_{\mathrm{PC}}=4.5 \mathrm{~Hz}, o-\mathrm{PPh}_{2}$, trans), $132.7 \sim 132.5$ (m, o- $-\mathrm{PPh}_{2}$ (trans to $\mathrm{Cl}$ ) and $o-\mathrm{PPh}_{2}$ (trans), partially overlapped), 132.4 (s, $\mathrm{C} \equiv \mathrm{CPh}), 131.8$ (t, $J_{\mathrm{PC}}=30.3 \mathrm{~Hz}$, ipso- $\mathrm{PPh}_{2}$, trans), 131.7 (s, C $\left.\equiv \mathrm{CPh}\right), 129.6$

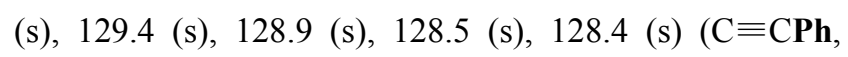
$p$ - $\left.\mathrm{PPh}_{2}\right), 128.8\left(\mathrm{t}, J_{\mathrm{PC}}=28.0 \mathrm{~Hz}\right.$, ipso- $\mathrm{PPh}_{2}$, trans $), 128.2$ (s, C $\equiv \mathrm{CPh}), 127.3\left(\mathrm{~d}, J_{\mathrm{PC}}=10.1 \mathrm{~Hz}, m-\mathrm{PPh}_{2}\right.$, trans to $\left.\mathrm{Cl}\right)$, $127.0\left(\mathrm{t}, J_{\mathrm{PC}}=4.4 \mathrm{~Hz}, m-\mathrm{PPh}_{2}\right.$, trans $), 126.6\left(\mathrm{t}, J_{\mathrm{PC}}=4.3\right.$ $\mathrm{Hz}, m-\mathrm{PPh}_{2}$, trans), 121.3 (s, ipso-PhC $\left.\equiv \mathrm{C}\right), 109.7$ (d, $J_{\mathrm{PC}}=8.3 \mathrm{~Hz}, \mathrm{Ph}_{2} \mathrm{PC} \equiv \mathbf{C}_{\beta} \mathrm{Ph}$, trans to $\left.\mathrm{Cl}\right), 109.5\left(\mathrm{t}, J_{\mathrm{PC}}=\right.$ $6.1 \mathrm{~Hz}, \mathrm{Ph}_{2} \mathrm{PC} \equiv \mathbf{C}_{\beta} \mathrm{Ph}$, trans $), 84.3\left(\mathrm{~d}, J_{\mathrm{PC}}=83.4 \mathrm{~Hz}\right.$, $\mathrm{Ph}_{2} \mathrm{PC}_{\alpha} \equiv \mathrm{CPh}$, trans to $\left.\mathrm{Cl}\right), 83.0\left(\mathrm{t}, J_{\mathrm{PC}}=47.9 \mathrm{~Hz}\right.$, $\mathrm{Ph}_{2} \mathrm{PC}_{\alpha} \equiv \mathrm{CPh}$, trans), $28.2 \quad\left(\mathrm{~s}, \mathrm{CH}_{3}\right), 22.8 \quad\left(\mathrm{~s}, \mathrm{CH}_{3}\right)$; ${ }^{31} \mathrm{P}\left\{{ }^{1} \mathrm{H}\right\} \quad \mathrm{NMR} \quad\left(\mathrm{CDCl}_{3}, 162 \mathrm{MHz}\right) \quad \delta:-28.1 \quad(\mathrm{t}$, unresolved), -28.6 (d, unresolved). Anal. calcd for $\mathrm{C}_{63} \mathrm{H}_{53} \mathrm{Cl}_{2} \mathrm{OsP}_{3}$ : C 65.17, H 4.50; found C 65.34, H 4.95.

3.2.4 $\mathrm{OsCl}_{3}\left(\equiv \mathrm{CCH}=\mathrm{CMe}_{2}\right)\left(\mathrm{Ph}_{2} \mathrm{PC} \equiv \mathrm{CPh}\right)_{2}(6)$ 的合 成

将 $\mathrm{OsCl}_{3}\left(\equiv \mathrm{CCH}=\mathrm{CMe}_{2}\right)\left(\mathrm{PPh}_{3}\right)_{2}$ (4) (500 mg, 0.56 $\mathrm{mmol}$ )和 $\mathrm{Ph}_{2} \mathrm{PC} \equiv \mathrm{CPh}(802 \mathrm{mg}, 2.80 \mathrm{mmol}$ )溶于甲苯 (25 $\mathrm{mL})$ 中, $90{ }^{\circ} \mathrm{C}$ 下加热搅拌反应, 并在反应过程中通过检 测反应液的 ${ }^{31} \mathrm{P}\left\{{ }^{1} \mathrm{H}\right\} \mathrm{NMR}$ 跟踪反应情况, 发现 $5 \mathrm{~h}$ 后反 应尚未完全且变得非常缓慢, 将反应液在减压条件下浓 缩至 $2 \mathrm{~mL}$, 加入乙醚/正己烷混和溶剂 $(V: V=3: 1) 20$ $\mathrm{mL}$ 并摚拌, 有大量紫红色沉淀生成, 过滤得到固体, 经 正己烷 $(5 \mathrm{~mL} \times 2)$ 洗涤后, 真空下抽干, 往所得的紫红色 固体中再加入 $\mathrm{Ph}_{2} \mathrm{PC} \equiv \mathrm{CPh}(802 \mathrm{mg}, 2.80 \mathrm{mmol})$, 并继 续在甲苯 $(25 \mathrm{~mL})$ 中于 $90{ }^{\circ} \mathrm{C}$ 下加热反应 $5 \mathrm{~h}$ 使得反应进
行完全, 重复上述处理步骤, 收集得到纯净的紫红色固 体 $467 \mathrm{mg}$, 产率 $89 \%$. m.p. $177 \sim 185{ }^{\circ} \mathrm{C}$ (分解); ${ }^{31} \mathrm{P}\left\{{ }^{1} \mathrm{H}\right\}$ NMR $\left(\mathrm{CDCl}_{3}, 202 \mathrm{MHz}\right) \delta:-28.2 ;{ }^{1} \mathrm{H}$ NMR $\left(\mathrm{CDCl}_{3}, 500 \mathrm{MHz}\right) \delta: 8.36 \sim 8.11(\mathrm{~m}, 8 \mathrm{H}, \mathrm{Ph}), 7.74 \sim 7.72$ (m, 4H, Ph), $7.55 \sim 7.32(\mathrm{~m}, 18 \mathrm{H}, \mathrm{Ph}), 4.21(\mathrm{~s}, 1 \mathrm{H}, \mathrm{CH}=$ ), $1.61\left(\mathrm{~s}, 3 \mathrm{H}, \mathrm{CH}_{3}\right), 1.10\left(\mathrm{~s}, 3 \mathrm{H}, \mathrm{CH}_{3}\right) ;{ }^{13} \mathrm{C}\left\{{ }^{1} \mathrm{H}\right\} \mathrm{NMR}$ $\left(\mathrm{CDCl}_{3}, 126 \mathrm{MHz}\right) \delta: 269.1\left(\mathrm{t}, J_{\mathrm{PC}}=12.5 \mathrm{~Hz}, \mathrm{Os} \equiv \mathrm{C}\right)$, $173.8\left[\mathrm{~s},=\mathbf{C}\left(\mathrm{CH}_{3}\right)_{2}\right], 133.7\left(\mathrm{t}, J_{\mathrm{PC}}=5.4 \mathrm{~Hz}\right.$, ortho $\left.-\mathrm{PPh}_{2}\right)$, 133.4 (s, ipso-PhC $\equiv \mathrm{C}), 133.0$ (s, $\mathbf{P h C} \equiv \mathrm{C}), 130.5$ (s, Ph$\mathrm{C} \equiv \mathrm{C}), 130.1(\mathrm{~s}$, para $-\mathrm{PhC} \equiv \mathrm{C}), 129.8\left(\mathrm{t}, J_{\mathrm{PC}}=31.2 \mathrm{~Hz}\right.$, ipso- $\left.\mathrm{PPh}_{2}\right), 128.4\left(\mathrm{~s}\right.$, para $\left.-\mathrm{PPh}_{2}\right), 127.8\left(\mathrm{t}, J_{\mathrm{PC}}=5.4 \mathrm{~Hz}\right.$, meta $\left.-\mathrm{PPh}_{2}\right), 121.3(\mathrm{~s}, \mathrm{CH}=), 112.7\left(\mathrm{t}, J_{\mathrm{PC}}=8.6 \mathrm{~Hz}\right.$, $\left.\mathrm{Ph}_{2} \mathrm{PC} \equiv \mathbf{C}_{\beta} \mathrm{Ph}\right), 79.3\left(\mathrm{t}, J_{\mathrm{PC}}=54.2 \mathrm{~Hz}, \mathrm{Ph}_{2} \mathrm{PC}_{\alpha} \equiv \mathrm{CPh}\right)$, $26.7\left(\mathrm{~s}, \mathrm{CH}_{3}\right), 24.4\left(\mathrm{~s}, \mathrm{CH}_{3}\right)$. Anal. calcd for $\mathrm{C}_{45} \mathrm{H}_{37} \mathrm{Cl}_{3}$ $\mathrm{OsP}_{2}$ : C 57.72, H 3.98, found C 57.98, H 4.21.

\section{3 配合物晶体结构测定}

挑选适当的化合物 3 的晶体, $173 \mathrm{~K}$ 下, 在 Rigaku R-Axis RAPID IP 单晶衍射仪上, 采用石墨单色化的 Mo $\mathrm{K} \alpha$ 射线 $(\lambda=0.071073 \mathrm{~nm})$ 收集数据. 衍射数据经过 multi-scan 吸收校正, 晶体结构采用 SHELXS-97 程序包 解析，对全部非氢原子坐标及其各向异性热参数进行全 矩阵最小二乘法修正. 化合物 3 的晶体数据存于英国剑 桥数据中心, CCDC 号为 1497480 .

辅助材料(Supporting Information) 化合物 $3 \sim 6$ 的 ${ }^{31} \mathrm{P}\left\{{ }^{1} \mathrm{H}\right\} 、{ }^{1} \mathrm{H}$ 和 ${ }^{13} \mathrm{C}\left\{{ }^{1} \mathrm{H}\right\}$ 核磁共振谱图. 这些材料可以免 费从本刊网站(http://sioc-journal.cn/)上下载.

\section{References}

[1] For selected general reviews on the chemistry of transition metal carbyne complexes see:

(a) Fischer, H.; Hofmann, P.; Kreissl, F. R.; Schrock, R. R.; Schubert, U.; Weiss, K. In Carbyne Complexes, VCH, Weinheim, Germany, 1988.

(b) Maya, A.; Hoffmeister, H. Adv. Organomet. Chem. 1991, 32, 227.

(c) Engel, P. F.; Pfeffer, M. Chem. Rev. 1995, 95, 2281.

(d) Schrock, R. R. J. Chem. Soc., Dalton Trans. 2001, 2541.

(e) Schrock, R. R. Chem. Rev. 2002, 102, 145.

(f) Shi, C.; Jia, G. Coord. Chem. Rev. 2013, 257, 666.

(g) Herndon, J. W. Coord. Chem. Rev. 2016, 317, 1;

[2] For selective reviews on alkyne metathesis see:

(a) Fürstner, A. Angew. Chem., Int. Ed. 2013, 52, 2794 and references cited therein.

(b) Schrock, R. R. Angew. Chem., Int. Ed. 2006, 45, 3748.

(c) Zhang, W.; Moore, J. S. Adv. Synth. Catal. 2007, 349, 93.

(d) Fürstner, A.; Davies, P. W. Chem. Commun. 2005, 2307.

(e) Bunz, H. F. Acc. Chem. Res. 2001, 34, 998.

[3] Selected recent examples of alkyne metathesis:

(a) on Kugelgen, S.; Bellone, D. E.; Cloke, R. R.; Perkins, W. S.; Fischer, F. R. J. Am. Chem. Soc. 2016, 138, 6234. 
(b) Wang, Q.; Yu, C.; Long, H.; Du, Y.; Jin, Y.; Zhang, W. Angew. Chem., Int. Ed. 2015, 54, 7550.

(c) Ralston, K. J.; Ramstadius, H. C.; Brewster, R. C.; Niblock, H. S.; Hulme, A. N. Angew. Chem., Int. Ed. 2015, 54, 7086.

(d) Haberlag, B.; Freytag, M.; Jones, P. G.; Tamm, M. Adv. Synth. Catal. 2014, 356, 1255.

(e) Lhermet, R.; Fürstner, A. Chem. Eur. J. 2014, 20, 13188.

(f) Li, S. T.; Schnabel, T.; Lysenko, S.; Brandhorst, K.; Tamm, M. Chem. Commun. 2013, 49, 7189.

(g) Yang, H.; Jin, Y.; Du, Y.; Zhang, W. J. Mater. Chem. A 2014, 2, 5986.

(h) Heppekausen, J.; Stade, R.; Goddard, R.; Fürstner, A. J. Am. Chem. Soc. 2010, 132, 11045.

(i) Weissman, H.; Plunkett, K. N.; Moore, J. S. Angew. Chem., Int. Ed. 2006, 45, 585 .

[4] For reviews on osmium carbyne complexes see:

(a) Bolaño, T.; Esteruelas, M. A.; Oñate, E. J. Organomet. Chem. 2011, 696, 3911.

(b) Jia, G. Coord. Chem. Rev. 2007, 251, 2167.

(c) Roper, W. R. In Transition Metal Carbyne Complexes, Ed.: Kreissl, F. R., Kluwer Academic: Dordrecht, The Netherlands, 1993, 155.

(d) Gallop, M. A.; Roper, W. R. Adv. Organomet. Chem. 1986, 25, 121.

[5] Selected recent examples of osmium carbyne complexes:

(a) Casanova, N.; Esteruelas, M. A.; Gulías, M.; Larramona, C.; Mascareñas, J. L.; Oñate, E. Organometallics 2016, 35, 91

(b) Zhou, X.; He, X.; Lin, J.; Zhuo, Q.; Chen, Z.; Zhang, H.; Wang, J.; Xia, H. Organometallics 2015, 34, 1742.

(c) Chen, J.; Huang, Z.-A.; Hua, Y.; Zhang, H.; Xia, H. Organometallics 2015, 34, 340.

(d) Buil, M. L.; Cardo, J. J. F.; Esteruelas, M. A.; Fernández, I.; Oñate, E. Organometallics 2015, 34, 547.

(e) Chen, J.; Zhang, C.; Xie, T.; Wen, T. B.; Zhang, H.; Xia, H. Organometallics 2013, 32, 3993.

(f) An, R.; Li, T.; Wen, T.-B. Chin. J. Org. Chem. 2013, 33, 1697 (in Chinese).

(安冉, 李亭, 温庭斌, 有机化学, 2013, 33, 1697.)

(g) Chen, J. X.; Sung, H. H. Y.; Williams, I. D.; Jia, G. Organometallics 2011, 30.

(h) Richter, B.; Werner, H. Organometallics 2009, 28, 5137.

(i) Bolaño, T.; Alba Collado, A.; Esteruelas, M. A.; Oñate, E. Organometallics 2009, 28, 2107.

(g) Castro-Rodrigo, R.; Esteruelas, M. A.; López, A. M.; Oñate, E. Organometallics 2008, 27, 3547.

(k) Castarlenas, R.; Esteruelas, M. A.; Lalrempuia, R.; Oliván, M.; Oñate, E. Organometallics 2008, 27, 795.

(1) Lee, J. H.; Pink, M.; Caulton, K. G. Organometallics 2006, 25, 802.

(m) Wen, T. B.; Hung, W. Y.; Zhou, Z. Y.; Lo, M. F.; Williams, I. D.; Jia, G. Eur. J. Inorg. Chem. 2004, 2837.

(n) Asensio, A.; Buil, M. L.; Esteruelas, M. A.; Oñate, E. Organometallics 2004, 23, 5787.

(o) Esteruelas, M. A.; López, A. M.; Oñate, E.; Royo, E. Organometallics 2004, 23, 3021 .

(p) Wen, T. B.; Zhou, Z. Y.; Lo, M. F.; Williams, I. D.; Jia, G. Organometallics 2003, 22, 5217.

(q) Barrio, P.; Esteruelas, M. A.; Oñate, E. Organometallics 2003, 22, 2472.

(r) Weberndörfer, B.; Henig, G.; Hockless, D. C. R.; Bennett, M. A.; Werner, H. Organometallics 2003, 22, 744.

(s) Castarlenas, R.; Esteruelas, M. A.; Oñate, E. Organometallics
2001, 20, 3283.

(t) Wen, T. B.; Cheung, Y. K.; Yao, J.; Wong, W. T.; Zhou, Z. Y.; Jia, G. Organometallics 2000, 19, 3803.

[6] (a) Buil, M. L.; Cardo, J. J. F.; Esteruelas, M. A.; Oñate, E. Organometallics 2016, 35, 2171.

(b) Buil, M. L.; Cardo, J. J. F.; Esteruelas, M. A.; Fernández, I.; Oñate, E. Organometallics 2014, 33, 2689.

(c) Bolaño, T.; Castarlenas, R.; Esteruelas, M. A.; Modrego, F. J.; Oñate, E. J. Am. Chem. Soc. 2005, 127, 11184.

(d) Barrio, P.; Esteruelas, M. A.; Oñate, E. J. Am. Chem. Soc. 2004, 126, 1946.

[7] (a) Jia, G. Organometallics 2013, 32, 6852 and references cited therein.

(b) Chen, J.; He, G.; Jia, G. Chin. J. Org. Chem. 2013, 33, 792 (in Chinese).

(陈江溪，何国梅，贾国成，有机化学，2013，33，792.)

(c) Chen, J.; Shi, C.; Sung, H. H. Y.; Williams, I. D.; Lin, Z.; Jia, G.. Angew. Chem., Int. Ed. 2011, 50, 7295.

(d) Hung, W. Y.; Liu, B.; Shou, W.; Wen, T. B.; Shi, C.; Sung, H. H.-Y.; Williams, I. D.; Lin, Z.; Jia, G. J. Am. Chem. Soc. 2011, 133, 18350 .

(e) He, G.; Zhu, J.; Hung, W. Y.; Wen, T. B.; Sung, H. H. Y.; Williams, I. D.; Lin, Z.; Jia, G. Angew. Chem. Int. Ed. 2007, 46, 9065.

(f) Hung, W. Y.; Zhu, J.; Wen, T. B.; Yu, K. P.; Sung, H. H.-Y.; Williams, I. D.; Lin, Z.; Jia, G. J. Am. Chem. Soc. 2006, 128, 13742. (g) Jia, G. Acc. Chem. Res. 2004, 37, 479.

[8] (a) Cao, X.-Y.; Zhao, Q.; Lin, Z.; Xia, H. Acc. Chem. Res. 2014, 47, 341.

(b) Chen, J.; Huang, Z.-A.; Lu, Z.; Zhang, H.; Xia, H. Chem. Eur. J. 2016, 22, 5363.

(c) Zhu, C.; Yang, Y.; Luo, M.; Yang, C.; Wu, J.; Chen, L.; Liu, G.; Wen, T.; Zhu, J.; Xia, H. Angew. Chem., Int. Ed. 2015, 54, 6181.

(d) Zhu, C.; Li, S.; Luo, M.; Zhou, X.; Niu, Y.; Lin, M.; Zhu, J.; Cao, Z.; Lu, X.; Wen, T.; Xie, Z.; Schleyer, P. V. R.; Xia, H. Nat. Chem. 2013, 5, 698.

(e) Liu, B.; Xie, H.; Wang, H.; Wu, L.; Zhao, Q.; Chen, J.; Wen, T. B.; Cao, Z.; Xia, H. Angew. Chem., Int. Ed. 2009, 48, 5461.

(f) Liu, B.; Wang, H.; Xie, H.; Zeng, B.; Chen, J.; Tao, J.; Wen, T. B.; Cao, Z.; Xia, H. Angew. Chem., Int. Ed. 2009, 48, 5430.

[9] Crabtree, R. H. The Organometallic Chemistry of the Transition Metals, 4th ed., John Wiley \& Sons, New York, 2005, pp. 309 340.

[10] (a) Mayr, A.; Asaro, M. F.; Kjelsberg, M. A.; Lee, K. S.; Van Engen, D. Organometallics 1987, 6, 432.

(b) Doyle, R. A.; Angelici, R. J. Organometallics 1989, 8, 2207.

(c) Blosch, L. L.; Abboud, K.; Boncella, J. M. J. Am. Chem. Soc. 1991, 113, 7066.

(d) Bastos, C. M.; Daubenspeck, N.; Mayr, A. Angew. Chem., Int. Ed. Engl. 1993, 32, 743.

(e) Giannini, L.; Solari, E.; Floriani, C.; Chiesi-Villa, A.; Rizzoli, C. J. Am. Chem. Soc. 1998, 120, 823.

(f) Bannwart, E.; Jacobsen, H.; Furno, F.; Berke, H. Organometallics 2000, 19, 3605.

[11] Boone, M. P.; Brown, C. C.; Ancelet, T. A.; Stephan, D. W. Organometallics 2010, 29, 4369.

[12] Esteruelas, M. A.; López, A. M.; Oliván, M. Coord. Chem. Rev. 2007, 251, 795.

[13] (a) Bolaño, T.; Castarlenas, R.; Esteruelas, M. A.; Oñate, E. Organometallics 2007, 26, 2037.

(b) Bolaño, T.; Castarlenas, R.; Esteruelas, M. A.; Oñate, E. J. Am. Chem. Soc. 2007, 129, 8850. 
(c) Buil, M. L.; Esteruelas, M. A.; Garcés, K.; Oñate, E. Organometallics 2009, 28, 5691.

[14] (a) Esteruelas, M. A.; González, A. I.; López, A. M.; Oñate, E. Organometallics 2003, 22, 414.

(b) Esteruelas, M. A.; González, A. I.; López, A. M.; Oñate, E. Organometallics 2004, 23, 4858.

(c) Castarlenas, R.; Esteruelas, M. A.; Oñate, E. Organometallics 2007, 26, 2129

(d) Buil, M. L.; Esteruelas, M. A.; Garcés, K.; Oliván, M.; Oñate, E. Organometallics 2008, 27, 4680 .

[15] Ozerov, O. V.; Watson, L. A.; Pink, M.; Caulton, K. G. J. Am. Chem. Soc. 2007, 129, 6003.

[16] Caulton, K. G. J. Organomet. Chem. 2001, 617 618, 56.

[17] (a) Ferrando, G.; Gérard, H.; Spivak, G. J.; Coalter III, J. N.; Huffman, J. C.; Eisenstein, O.; Caulton, K. G. Inorg. Chem. 2001, 40,6610 .

(b) Ferrando-Miguel, G.; Gérard, H.; Eisenstein, O.; Caulton, K. G. Inorg. Chem. 2002, 41, 6440.

(c) Ferrando, G.; Coalter, J. N.; Gerard, H.; Huang, D.; Eisenstein, O.; Caulton, K. G. New J. Chem. 2003, 27, 1451.

(d) Grünwald, G.; Gevert, O.; Wolf, J.; González-Herrero, P.; Werner, H. Organometallics 1996, 15, 1960.

[18] (a) Espuelas, J.; Esteruelas, M. A.; Lahoz, F. J.; Oro, L. A.; Ruiz, N. J. Am. Chem. Soc. 1993, 115, 4683.

(b) Collado, A.; Esteruelas, M. A.; López, F.; Mascareñas, J. L.; Oñate, E.; Trillo, B. Organometallics 2010, 29, 4966.

(c) Collado, A.; Esteruelas, M. A.; Oñate, E. Organometallics 2011, 30, 1930.

[19] Spivak, G. J.; Coalter, J. N.; Olivan, M.; Eisenstein, O.; Caulton, K. G. Organometallics 1998, 17, 999.

[20] (a) Vougioukalakis, G. C.; Grubbs, R. H. Chem. Rev. 2010, 110, 1746.

(b) Grubbs, R. H. Tetrahedron 2004, 60, 7117.

(c) Trnka, T. M.; Grubbs, R. H. Acc. Chem. Res. 2001, 34, 18.

[21] Grubbs has reported the reaction of $\mathrm{OsCl}_{2}\left(\mathrm{PPh}_{3}\right)_{3}$ with diphenylcyclopropene to afford $\mathrm{OsCl}_{2}\left(=\mathrm{CHCH}=\mathrm{CPh}_{2}\right)\left(\mathrm{PPh}_{3}\right)_{2}$, but no experimental evidence has been given, see:

(a) Nguyen, S. T.; Johnson, L. K.; Grubbs, R. H.; Ziller, J. W. J. Am. Chem. Soc. 1992, 114, 3974.

(b) Grubbs, R. H.; Schwab, P.; Nguyen, S. T. WO 9706185, 1997 [Chem. Abstr. 1997, 126, 238816].

[22] Wilhelm, T. E.; Belderrain, T. R.; Brown, S. N.; Grubbs, R. H.
Organometallics 1997, 16, 3867.

[23] (a) Volland, M. A. O.; Rominger, F.; Eisenträger, F.; Hofmann, P. J. Organomet. Chem. 2002, 641, 220.

Related syntheses of a series of ruthenium alkenylcarbenes $\mathrm{RuCl}_{2}\left(=\mathrm{CHCH}=\mathrm{CR}^{1} \mathrm{R}^{2}\right)(\mathrm{dtbpm})$ with a chelating ligand bis(di-tert-butyl-phosphanyl)methane $t$ - $\mathrm{Bu}_{2} \mathrm{PCH}_{2} \mathrm{P} t-\mathrm{Bu}_{2} \quad$ (dtbpm) from the reactions of a dinuclear ruthenium hydride $\left[\mathrm{RuH}\left(\mu_{2}-\mathrm{Cl}\right)\right.$ $(\mathrm{dtbpm})]_{2}$ with propargylic chlorides $\mathrm{HC} \equiv \mathrm{CC}(\mathrm{Cl}) \mathrm{R}^{1} \mathrm{R}^{2}$ have also been reported by Hofmann:

(b) Hansen, S. M.; Rominger, F.; Metz, M.; Hofmann, P. Chem.Eur. J. 1999, 5, 557.

(c) Hansen, S. M.; Volland, M. A. O.; Rominger, F.; Eisenträger, F.; Hofmann, P. Angew. Chem., Int. Ed. 1999, 38, 1273.

[24] Amoroso, D.; Snelgrove, J. L.; Conrad, J. C.; Drouin, S. D.; Yap, G. P. A.; Fogg, D. E. Adv. Synth. Catal. 2002, 344, 757.

[25] Werner, H.; Jung, S.; Weberndörfer, B.; Wolf, J. Eur. J. Inorg. Chem. 1999, 1999, 951.

[26] Ferrando, G.; Caulton, K. G. Inorg. Chem. 1999, 38, 4168.

[27] (a) Bustelo, E.; Jiménez-Tenorio, M.; Mereiter, K.; Puerta, M. C.; Valerga, P. Organometallics 2002, 21, 1903.

(b) Esteruelas, M. A.; Oliván, M.; Oñate, E. Organometallics 1999, 18, 2953.

(c) Crochet, P.; Esteruelas, M. A.; López, A. M.; Martinez, M. P.; Oliván, M.; Oñate, E.; Ruiz, N. Organometallics 1998, 17, 4500.

[28] (a) Bruce, M. I. Chem. Rev. 1991, 91, 197.

(b) Bruce, M. I. Chem. Rev. 1998, 98, 2797

[29] (a) Wen, T. B.; Yang, S. Y.; Zhou, Z. Y.; Lin, Z.; Lau, C. P.; Jia, G. Organometallics 2000, 19, 3757.

(b) Wen, T. B.; Zhou, Z. Y.; Jia, G. Angew. Chem., Int. Ed. 2001, 40, 1951.

(c) Wen, T. B.; Hung, W. Y.; Sung, H. H. Y.; Williams, I. D.; Jia, G. J. Am. Chem. Soc. 2005, 127, 2856.

(d) Wen, T. B.; Lee, K.-H.; Chen, J.; Hung, W. Y.; Bai, W.; Li, H.; Sung, H. H. Y.; Williams, I. D.; Lin, Z.; Jia, G. Organometallics 2016, 35, 1514.

[30] Collado, A.; Esteruelas, M. A.; López, F.; Mascareñas, J. L.; Oñate, E.; Trillo, B. Organometallics 2010, 29, 4966.

[31] Spivak, G. J.; Caulton, K. G. Organometallics 1998, 17, 5260.

[32] Hoffmann, P. R.; Caulton, K. G. J. Am. Chem. Soc. 1975, 97, 4221.

[33] Kondoh, A.; Yorimitsu, H.; Oshima, K. J. Am. Chem. Soc. 2007, $129,4099$.

(Qin, X.) 